\title{
DNA polymerase gamma (Poly) deficiency triggers a selective mTORC2 prosurvival autophagy response via mitochondria- mediated ROS signaling
}

\author{
Sanjit K. Dhar ${ }^{1} \cdot$ Vasudevan Bakthavatchalu $^{2} \cdot$ Bithika Dhar $^{1} \cdot$ Jing Chen $^{3} \cdot$ Izumi Tadahide $^{1} \cdot$ Haining Zhu $^{3} \cdot$ \\ Tianyan $\mathrm{Gao}^{3}$ - Daret K. St. Clair ${ }^{1}$
}

Received: 6 November 2017 / Revised: 30 April 2018 / Accepted: 11 June 2018 / Published online: 23 July 2018

(c) The Author(s) 2018. This article is published with open access

\begin{abstract}
Autophagy is a highly regulated evolutionarily conserved metabolic process induced by stress and energy deprivation. Here, we show that DNA polymerase gamma (Pol $\gamma$ ) deficiency activates a selective prosurvival autophagic response via mitochondria-mediated reactive oxygen species (ROS) signaling and the mammalian target of rapamycin complex 2 (mTORC2) activities. In keratinocytes, Pol $\gamma$ deficiency causes metabolic adaptation that triggers cytosolic sensing of energy demand for survival. Knockdown of Pol $\gamma$ causes mitochondrial stress, decreases mitochondrial energy production, increases glycolysis, increases the expression of autophagy-associated genes, and enhances AKT phosphorylation and cell proliferation. Deficiency of Pol $\gamma$ preferentially activates mTORC2 formation to increase autophagy and cell proliferation, and knocking down Rictor abrogates these responses. Overexpression of Rictor, but not Raptor, reactivates autophagy in Pol $\gamma$-deficient cells. Importantly, inhibition of ROS by a mitochondria-selective ROS scavenger abolishes autophagy and cell proliferation. These results identify Rictor as a critical link between mitochondrial stress, ROS, and autophagy. They represent a major shift in our understanding of the prosurvival role of the mTOR complexes and highlight mitochondriamediated ROS as a prosurvival autophagy regulator during cancer development.
\end{abstract}

\section{Introduction}

DNA polymerase gamma (Pol $\gamma$ ) is a nuclear-encoded, mitochondrially active DNA replication and repair enzyme that is essential for the survival of eukaryotic life [1-5]. Pol $\gamma$ homozygous knockout in mice causes embryonic lethality due to an early developmental defect associated with severe depletion of mitochondrial DNA (mtDNA) [6].

Electronic supplementary material The online version of this article (https://doi.org/10.1038/s41388-018-0404-z) contains supplementary material, which is available to authorized users.

Daret K. St. Clair

dstc100@uky.edu

1 Department of Toxicology and Cancer Biology, University of Kentucky, Lexington, KY 40536, USA

2 Division of Comparative Medicine, Massachusetts Institute of Technology, Cambridge, MA 02139, USA

3 Department of Molecular and Cellular Biochemistry, University of Kentucky, Lexington, KY 40536, USA
Because mtDNA encodes 13 proteins that, along with over 85 nuclear-encoded proteins, assemble into the oxidative phosphorylation system [7, 8], maintenance of mtDNA levels and integrity is critically important for mitochondrial energy production.

We have previously shown that Pol $\gamma$ becomes nitrated and is subsequently inactivated in UV-induced skin carcinogenesis [9], but the mechanisms by which this occurs are not well characterized. UV irradiation of skin cells triggers the production of nitric oxide, which, when combined with superoxide, forms peroxynitrite $\left(\mathrm{OONO}^{-}\right)$, a very potent oxidant species that modifies the tyrosine residues of proteins. Such modifications are regarded as a marker for nitrative stress [10], and Pol $\gamma$ is highly susceptible to peroxynitrite attack due to the presence of 31 tyrosine residues in its catalytic subunit, including the two highly conserved tyrosines in its active site [11].

The downstream effects of carcinogenic inactivation of Pol $\gamma$ are the object of ongoing investigation. Several lines of evidence have demonstrated that the oxidative stress leading to DNA damage provokes organelle defects which activate autophagic recycling, resulting in either cell death or 
survival [12]. In the context of many cellular stressors, ranging from hypoxia to DNA damage, autophagy constitutes a key prosurvival response, allowing adaptation to unfavorable conditions [13-15]. Autophagy facilitates the turnover of damaged organelles, including the mitochondria. This process occurs in cancer cells, leading to cell growth and proliferation by elevating glycolysis, which is also known as Warburg effect [16]. Because of the role of Pol $\gamma$ in the maintenance of mtDNA, we propose a link between Pol $\gamma$ activity, mitochondrial integrity, ROS, and autophagy. In this study, we provide evidence that loss of Pol $\gamma$ activity causes mitochondrial stress, leading to metabolic reprogramming, and autophagy via the mammalian target of rapamycin complex 2 (mTORC2).

\section{Results}

\section{Nitration of Poly and its effect on enzymatic activity}

It has been shown that UVB increases peroxynitrite generation [17, 18]. To elucidate whether and how UVB treatment causes Pol $\gamma$ nitration, we exposed primary human epidermal keratinocytes or JB6 cells to UVB radiation and used a 3-nitrotyrosine antibody to detect nitrated Pol $\gamma$. The nitration of Pol $\gamma$ was detected in both primary human epidermal keratinocytes and JB6 cells following UVB radiation (Fig. 1a, b). Further, reverse immunoprecipitation was performed using Pol $\gamma$ antibody and the nitration of Pol $\gamma$ was confirmed by western blotting using 3-nitrotyrosine antibody after UVB treatment (Fig. 1a bottom panel). To verify the nitration-mediated inactivation of the enzymatic activity upon UVB treatment, we measured Pol $\gamma$ activity using isolated mitochondria. Our data show that Poly activity in human and murine keratinocytes is significantly decreased following UVB treatment (Fig. 1c, d). These results support our previous findings and confirm that Pol $\gamma$ becomes nitrated after UVB irradiation in human and murine keratinocytes and consequently loses enzymatic activity.

\section{Identification of nitration site in Polp}

To precisely identify the nitrated amino acid residue(s) in Pol $\gamma$, we purified full-length recombinant mouse Pol $\gamma$ (Fig. 1e) and exposed it to authentic peroxynitrite in vitro. The nitration of Pol $\gamma$ by peroxynitrite was confirmed by immunoprecipitation using a 3-nitrotyrosine antibody followed by western blotting against Pol $\gamma$ (Fig. 1f). A Pol $\gamma$ reverse transcriptase activity assay was performed using purified protein following peroxynitrite treatment. The activity of peroxynitrite-treated Pol $\gamma$ was significantly reduced compared to the saline-treated protein (Fig. 1g).
To identify the specific nitrotyrosine modification of Pol $\gamma$, we digested the purified peroxynitrite-treated Pol $\gamma$ protein with trypsin and analyzed the peptide mixture by mass spectrometry. Nitrated peptides were identified using liquid chromatography electrospray ionization (LCESI)MS/MS analysis. It has been demonstrated that introduction of a nitro $\left(\mathrm{NO}_{2}\right)$ group onto a tyrosine results in a difference in $\mathrm{m} / \mathrm{z}$ ratio of +45 [19]. Figure $1 \mathrm{~h}$ shows the LCESI-MS/ MS spectra of the tryptic peptides of untreated Pol $\gamma$ and peroxynitrite-treated Pol $\gamma$. In the MS/MS spectra, the tyrosine contained in peptide y5 has an $\mathrm{m} / \mathrm{z}$ of 581.42 in the untreated sample, whereas peroxynitrite-treated peptide y 5 has an $\mathrm{m} / \mathrm{z}$ ratio of 626.44 . This difference in molecular mass indicates the nitration of a single tyrosine residue in Pol $\gamma$, and the MS/MS spectra identified this peptide as containing tyrosine 964 in the catalytic domain of the protein. Interestingly, Y964 is well conserved in a variety of species ranging from humans to Saccharomyces (Supplementary Figure S1).

\section{Poly deficiency causes mitochondrial DNA damage and mitochondrial dysfunction}

Pol $\gamma$ plays a pivotal role in protecting the mitochondrial genome from oxidative damage [20]. To demonstrate this role of Pol $\gamma$, we generated stable Pol $\gamma$-knockout cell lines using CRISPR-mediated gene editing technology (Fig. 2a). Poly-knockout PCRISPR cells were selected by using puromycin selection antibiotics. Among several stable positive clones, complete knockdown PCRISPR clones were selected and propagated. To rule out the off-target effect of the CRISPR method, we assessed the mitochondrial resident MnSOD proteins by western blotting. Furthermore, we detected Pol $\gamma$ in PCRISPR cells by overexpressing wildtype and mutant Pol $\gamma$ proteins (Figure S5). The expression of Pol $\gamma$ was determined by western blotting and the activity by primer extension using isolated mitochondria from Pol $\gamma$ knockout (PCRISPR) cells. As shown in Fig. 2b, c, both Pol $\gamma$ protein levels and Pol $\gamma$ activity were diminished in PCRISPR cells.

To investigate the impact of Pol $\gamma$ deficiency on mtDNA damage, we amplified a $6.9 \mathrm{~kb}$ DNA fragment and $0.2 \mathrm{~kb}$ DNA fragment (long and short amplicons, respectively) using mitochondrial gene-specific primers. The amplified mtDNA was quantified and normalized against nuclear DNA to assess the relative changes in the mitochondrial amplicons. Further, we assessed the relative levels of DNA damage relative to the levels of mitochondrial mass [21]. To assess the mitochondrial mass, we stained both control and PCRISPR cells using mitochondria-specific cardiolipin binding dye NAO (10-nonylacridine orange bromide) followed by flow cytometry. The NAO uptake in control and PCRISPR was used to normalize the mitochondrial DNA 


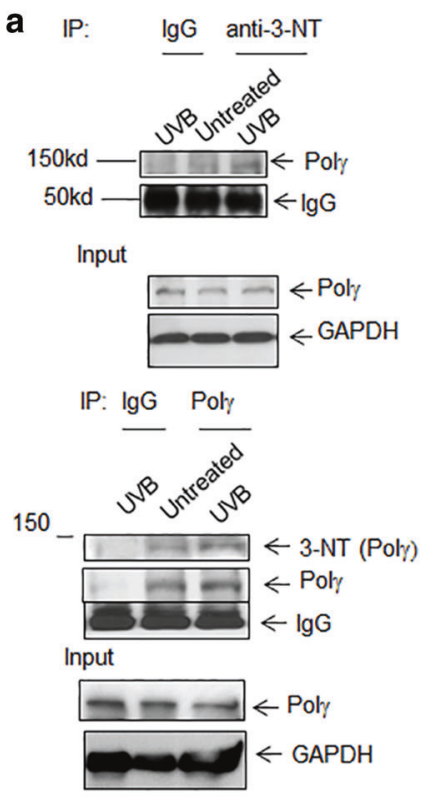

b
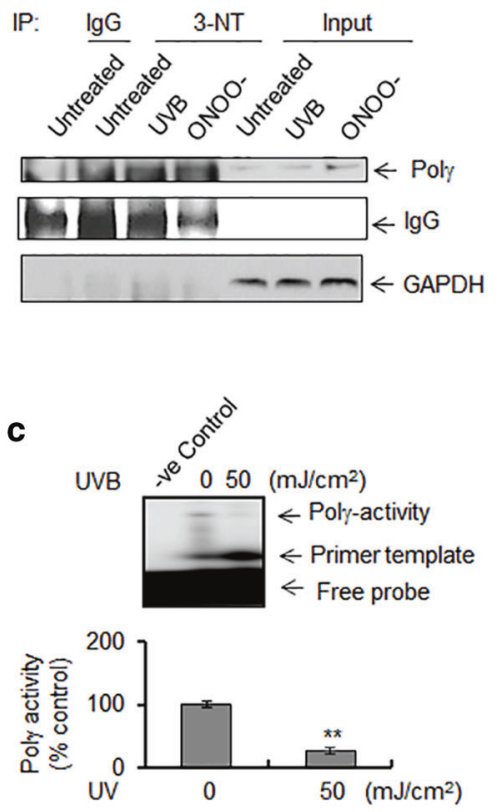

d

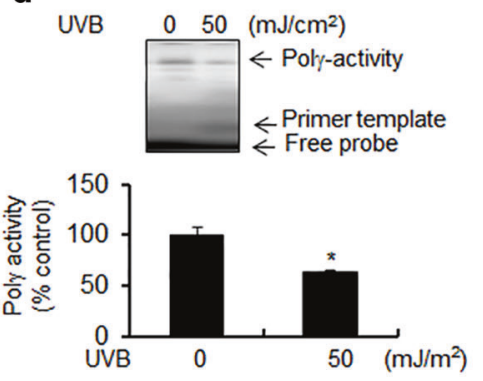

e

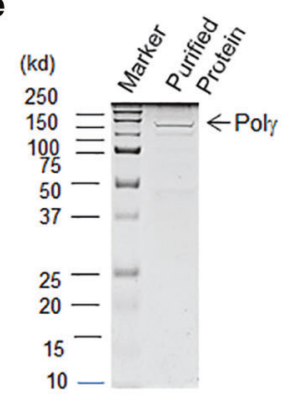

f

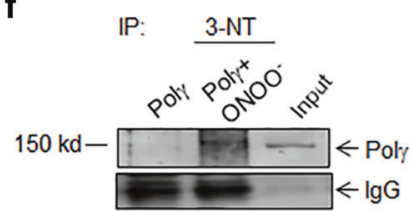

g

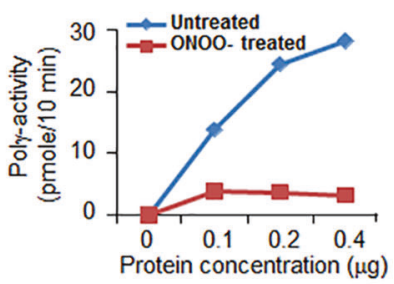

h
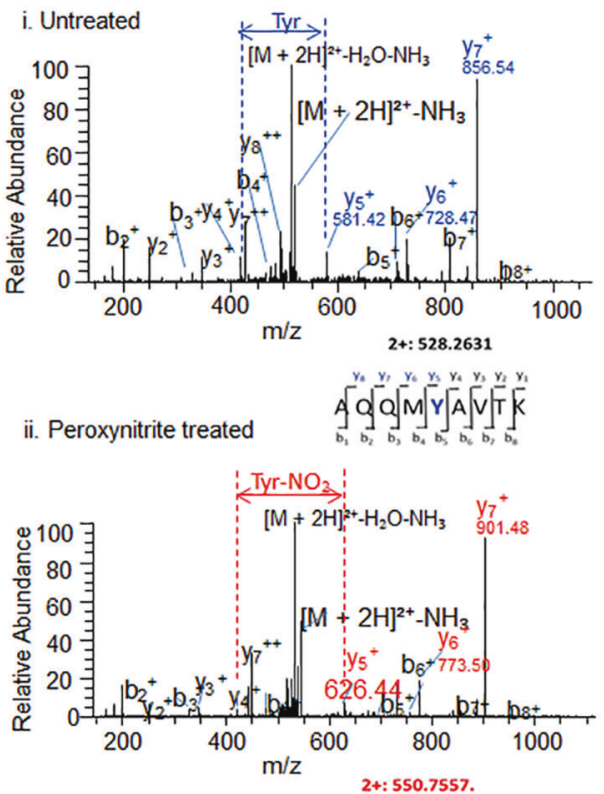

50

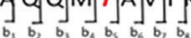

$Y=\operatorname{Tyr}-\mathrm{NO}_{2}(+44.9851 \mathrm{Da})$ damage in control and PCRISPR cells, respectively. The data from PCR analysis shown in Fig. 2d demonstrate that the amount of long-amplicon mtDNA relative to shortamplicon mtDNA is significantly lower in Pol $\gamma$-deficient 
Fig. 1 Pol $\gamma$ nitration and activity. a Detection of Pol $\gamma$ nitration after UVB irradiation $\left(50 \mathrm{~mJ} / \mathrm{cm}^{2} \times 1 \mathrm{~h}\right)$ in human primary epidermal keratinocytes using 3-nitrotyrosine immunoprecipitation followed by western blotting with Pol $\gamma$ antibody or Pol $\gamma$ antibody-mediated immunoprecipitation followed by western blotting with 3nitotyrosine antibody. Both $\mathrm{IgG}$ and inputs were provided as loading control. b Detection of Pol $\gamma$ nitration by immunoprecipitation after UVB treatment $\left(50 \mathrm{~mJ} / \mathrm{cm}^{2}\right)$ in JB6 cells using 3-nitrotyrosine antibody with exposure to authentic peroxynitrite $\left(\mathrm{ONOO}^{-}\right)$as a positive control. Both IgG and inputs were provided as loading control. c Pol $\gamma$ activity in human keratinocytes with or without UVB treatment was detected by $20 \%$ acrylamide/7 M urea gel electrophoresis and autoradiography following primer extension using mtDNA-specific primers (see Materials and methods). d Pol $\gamma$ activity in JB6 cells was detected as described in panel (c). e SDS-PAGE of purified recombinant mouse Pol $\gamma$ protein and visualization by Coomassie brilliant blue staining. f Purified Pol $\gamma$ proteins were immunoprecipitated with 3nitrotyrosine antibody before and after treatment with peroxynitrite $(250 \mu \mathrm{M})$. Pol $\gamma$ proteins were detected by western blotting using an anti-Pol $\gamma$ antibody. Both IgG and inputs were used as loading control. g Activity of purified Poly protein was measured by dTTP incorporation in the presence or absence of peroxynitrite as described in the Materials and methods. h Nitration of tyrosine residues was detected by mass spectrometry. Each peak in the MS/MS spectrum represents relative abundance of peptide fragment and the tyrosine residues of the peptide fragment are shown under each graph. For all quantifications (c, d), each data point represents the mean \pm SD of three individual samples. For all panels, representative figures from three repeated experiments are shown. Statistical analysis was performed using $t$ tests. Statistical significance is indicated by asterisks: $* p<0.05$ and $* * p<0.01$

CRISPR cells as compared to control cells. The observed number of mtDNA lesions per $10 \mathrm{~kb}$ was greater in Pol $\gamma$ deficient CRISPR cells than in control cells, indicating an increase in mtDNA damage (Fig. 2e).

To determine how Pol $\gamma$ deficiency affects mitochondrial function, we measured cellular bioenergetics. The data presented in Fig. $2 \mathrm{f}$ show that oxygen consumption was decreased in PCRISPR cells compared to controls, resulting in a decrease in basal respiration, ATP-linked respiration, maximum capacity, and reserve respiratory capacity. Conversely, the extracellular acidification rate was increased in PCRISPR cells as compared to controls, resulting in increases in glycolysis, glycolytic capacity, and glycolytic reserve (Fig. 2g). PCRISPR cells also exhibited an increase in lactate levels compared to control cells (Supplementary Figure S2). These results suggest that the deficiency of Pol $\gamma$ in PCRISPR cells causes an increase in mitochondrial stress, which leads to the impairment of oxidative phosphorylation and a shift toward glycolysis.

Mitochondrial ATP production is driven by electrons being passed along the electron transport chain (ETC), with a considerable number leaking as superoxide radicals/ anions $\left(\mathrm{O}_{2}{ }^{-}\right)$during the process. To determine if Pol $\gamma$ deficiency contributes to the generation of $\mathrm{O}_{2}{ }^{--}$in mitochondria, we used fluorometric analysis, with positive and negative controls, to quantify $\mathrm{O}_{2}{ }^{--}$in Pol $\gamma$-deficient
PCRISPR and control JB6 cells. Mitochondrial superoxide anion was measured using MitoSox Red, a fluorogenic dye that specifically targets mitochondria in live cells. The relative mitochondrial mass was used to normalize MitoSox Red to quantify mitochondrial superoxide anion, with rotenone as a positive control. The levels of superoxide radicals in Pol $\gamma$-deficient PCRISPR cells were significantly higher than the levels in control cells (Fig. 2h). Rotenone, an inhibitor of ETC complex I, is able to inhibit mitochondrial oxidative phosphorylation, leading to generation of high levels of superoxide radicals. The results demonstrate a higher level of $\mathrm{O}_{2}{ }^{-}-$in Pol $\gamma$-deficient cells exposed to rotenone (Fig. 2h).

\section{Deficiency of Poly activates autophagy}

As autophagy is critical for old and damaged organelles, we investigated the role of Pol $\gamma$ in autophagy. Microtubuleassociated protein light chain 3 (LC3) is an abundant cytoplasmic protein that is cleaved and lipidated during initiation of autophagy (forming LC3B or LC3 II) and is incorporated into autophagosomal membranes in a punctate pattern [22]. LC3B is a downstream indicator of the autophagic pathway and participates in autophagosome formation and maturation [23].

To test the role of Poly in autophagy, LC3-GFP expression vectors were transfected into Pol $\gamma$-deficient CRISPR cells. We then determined the levels of LC3 punctation and LC3 II formation by fluorescence microscopy and western blotting, respectively. Figure 3 a shows that LC3 punctation was significantly increased in PCRISPR cells as compared to control cells. Consistent with this finding, the endogenous level of LC3 II formation was increased in PCRISPR cells (Fig. 3b).

We further assessed the critical upstream autophagy regulators beclin 1 and Atg7. Beclin 1 is responsible for initiation of autophagosome formation [24, 25]. Consistent with the LC3 II formation, beclin 1 was significantly increased in PCRISPR cells as compared to control cells (Fig. 3b). The autophagic function of Beclin 1 is regulated by its phosphorylation at S234 and S295 [26]. The Beclin 1 phosphorylation was not altered in PCRISPR cells as compared to control (Fig. 3b). The autophagy initiator ATG7 was also significantly increased in PCRISPR cells as compared to controls (Fig. 3b). Consistent with an increase in autophagy, the level of SQSTM1 (p62) protein, which recognizes toxic cellular waste, was reduced in PCRISPR cells.

Autophagy flux was assessed to confirm the increase of autophagy in Pol $\gamma$-deficient CRISPR cells as detected by western blotting and fluorescence microscopy (Fig. 3c, d). The results show that higher levels of LC3 II in PCRISPR cells, as compared to control cells, can be suppressed by 


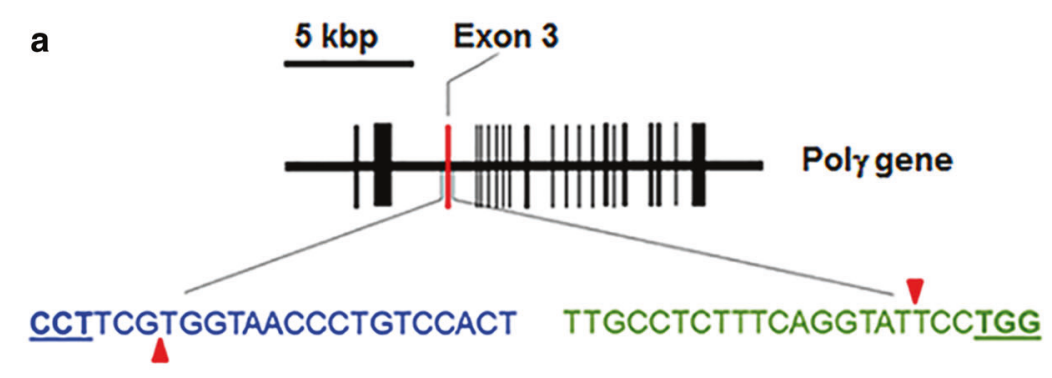

b
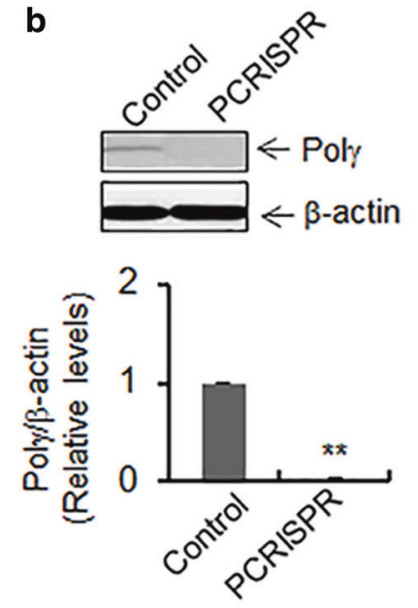

c
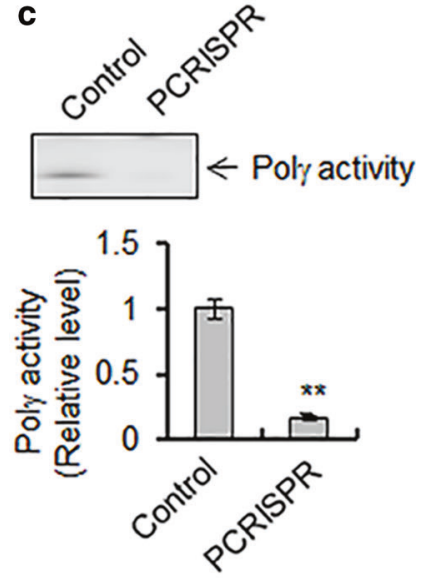

d

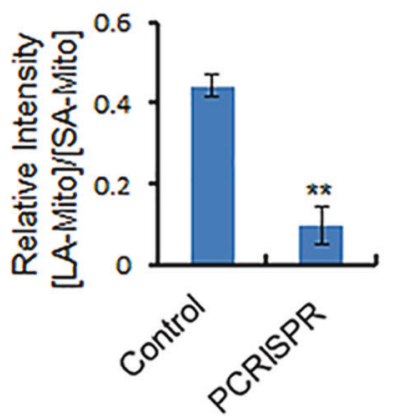

e

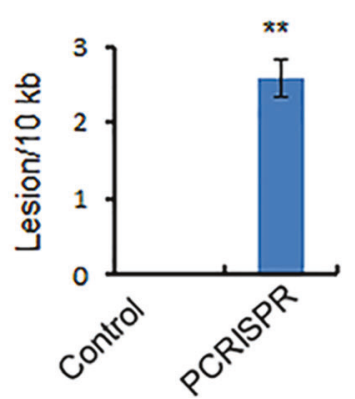

高
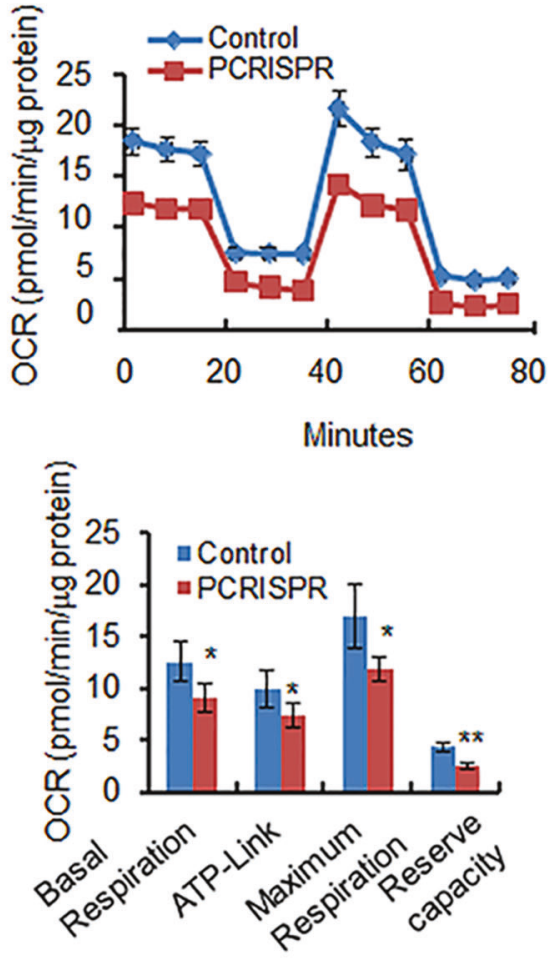

g
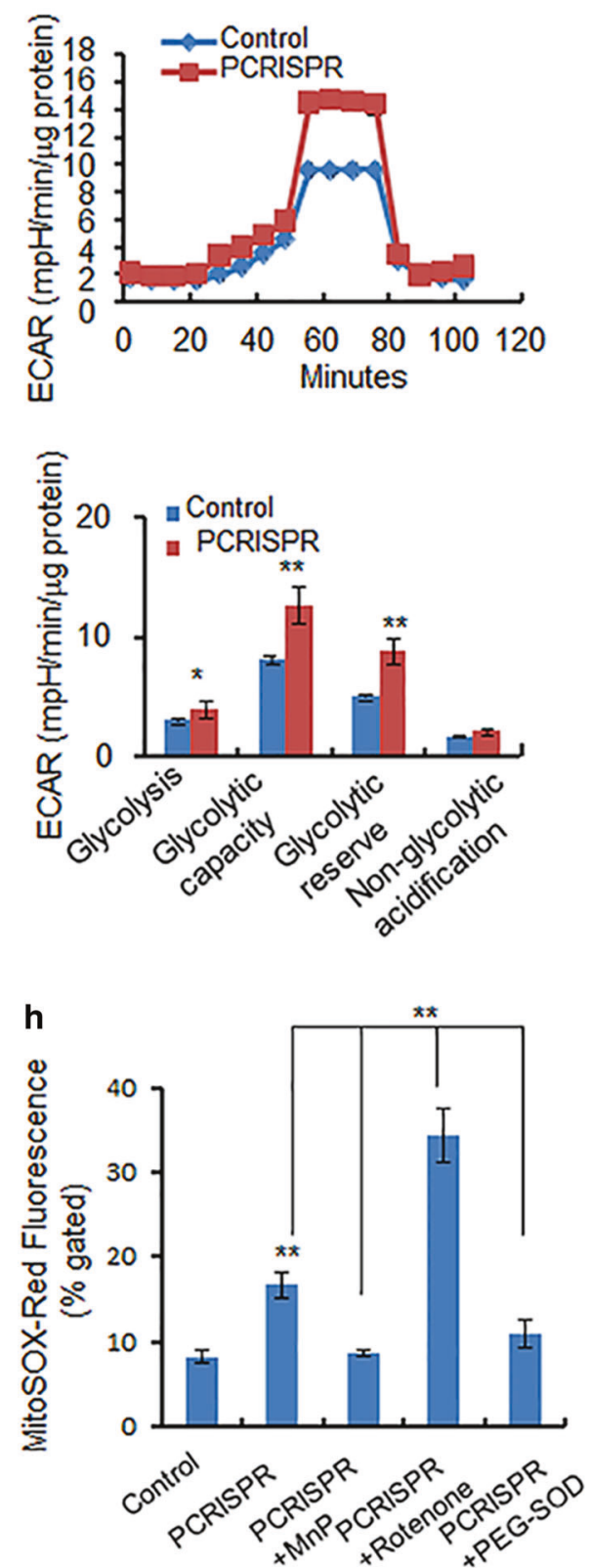

pretreatment with the ROS inhibitor $\mathrm{MnP}$ or the $\mathrm{PI} 3 \mathrm{k}$ inhibitor 3-MA. On the other hand, Pol $\gamma$-deficiency-mediated increases of LC3 II levels and LC3 punctation were further increased after treatment with bafilomycin for $24 \mathrm{~h}$ as compared to PCRISPR alone. These results confirm that a deficiency of Pol $\gamma$ increases autophagy. 
Fig. 2 Effects of Pol $\gamma$ suppression on mitochondria. a The mouse Pol $\gamma$ gene structure in chromosome 7 is shown schematically. The vertical bars indicate the exons. The exon 3 targeted by CRISPR is highlighted in red. Two 20-nucleotide guide sequences of CRISPR are shown, in blue and in green. The pam sequences are underlined, and red triangles indicate the incision sites by Cas9n. b Pol $\gamma$ protein levels were measured by western blotting in PCRISPR JB6 clones. $\mathbf{c}$ Pol $\gamma$ activity in mitochondria isolated from PCRISPR cells was detected by primer extension followed by autoradiography (see Materials and methods). d The PCR product ratio of long-amplicon (LA) mtDNA to shortamplicon (SA) mtDNA in control and Pol $\gamma$-deficient PCRISPR cells which is normalized by mitochondrial mass. e The number of DNA lesions per $10 \mathrm{~kb}$ mtDNA in Pol $\gamma$-deficient PCRISPR cells following normalization with mitochondrial mass. Note that the mitochondrial masses of control and PCRISPR cells were obtained by staining the cells with NAO, a mitochondria-specific dye, followed by flow cytometry. f Mitochondrial oxygen consumption was measured as described in Materials and Methods. OCR oxygen consumption rate. $\mathbf{g}$ Glycolysis was measured as described in Materials and Methods. ECAR extracellular acidification rate. $\mathbf{h}$ The level of mitochondrial reactive oxygen was measured by quantifying the MitoSox Red fluorescence in Pol $\gamma$-deficient PCRISPR cells in the presence and absence of $\mathrm{MnP}\left(\mathrm{MnTnBuOE}-2-\mathrm{PyP}^{5+}\right.$ ), with positive and negative controls. The mean fluorescence intensity of MitoSox Red was determined using flow cytometry. The concentration of cellular superoxide was estimated by quantification of fluorescence intensity. Rotenone was used as a positive control for generation of ROS. PEGSOD (Superoxide dismutase-polyethylene glycol from bovine erythrocytes) was also used as a control to remove superoxide generated by MitoSox Red. The fluorescence intensity of MitoSox Red is normalized by total mitochondrial mass. In all bar graphs and line graphs, each data point represents the mean \pm SD of three individual samples. Each experiment was repeated at least three times and statistical analysis was performed using $t$ tests for two groups or one-way ANOVA analysis and Bonferroni's post-test for multiple-group comparisons. Statistical significance is indicated by asterisks: $* p<0.05$ and $* * p<$ 0.01

\section{Mutation of Y964 inactivates Poly and triggers autophagy}

As shown in Fig. 1h, using mass spectrometric analysis, we identified the tyrosine nitration at the Y964 site in the catalytic domain of Poly. To examine the significance of tyrosine nitration in Pol $\gamma$, we performed site-directed mutagenesis to generate a Pol $\gamma$-Y964F mutant. We then overexpressed wild-type Pol $\gamma$ or the Y964F mutant in Pol $\gamma$ deficient CRISPR cells as well as Poly-knockdown cells and compared the reverse transcriptase activity of Poly. Equal overexpression of both wild-type and mutant proteins following transfection were confirmed by western blotting (Fig. 4a, b). Overexpression of Pol $\gamma$ in PCRISPR cells significantly increased the enzymatic activity as compared with PCRISPR controls (Fig. 4a). Overexpression of mutant Pol $\gamma$ increased the Pol $\gamma$ activity about half as much as overexpression of the wild-type protein, suggesting that the Y964F mutation causes a loss of Poly activity (Fig. 4a). Similar results were observed in JB6 cells with knockdown of endogenous Pol $\gamma$ by siRNA (Fig. 4b).
Next, we determined how the expression of wild-type and mutant Pol $\gamma$ affects the level of LC3 II formation observed in Poly-deficient cells. Consistent with the results for Pol $\gamma$ activity, overexpression of wild-type Pol $\gamma$ suppressed the LC3 II formation which was enhanced in Polydeficient cells (Fig. 4c). However, overexpression of mutant Pol $\gamma$ was not able to suppress autophagic LC3 II formation (Fig. 4c). These results show that the mutation of Y964 in the catalytic domain significantly suppresses the enzymatic activity of Pol $\gamma$ and consequently limits its ability to prevent autophagy.

To examine the biological relevance of Poly deficiency, we overexpressed wild-type and mutant Pol $\gamma$ in JB6 cells and assessed LC3 II formation after UVB treatment. The autophagic LC3 II formation was significantly increased upon UVB radiation and was attenuated by wild-type Poly overexpression but not mutant Pol $\gamma$ overexpression (Figure S4).

\section{Deficiency of Poly activates mTOR/AKT pathways}

How Pol $\gamma$ deficiency leads to autophagy is not clear. We hypothesized that a deficiency of Pol $\gamma$ that causes aberrant mitochondrial function could activate stress signals that affect the mTOR signaling pathway. To test this hypothesis, we investigated mTOR activation in both PCRISPR cells and stable Pol $\gamma$-knockdown cells. As shown in Fig. 5, the phosphorylation of mTOR (2481) was significantly increased in Pol $\gamma$-deficient CRISPR cells (Fig. 5a) as well as Pol $\gamma$-knockdown cells (Fig. 5b) as compared to the respective controls. Consistent with mTOR phosphorylation, AKT phosphorylation was also increased significantly. A remarkable increase in Rictor, which is a component of mTORC2, was observed in PCRISPR as well as in Pol $\gamma$-knockdown cells. However, no change in level occurred in Raptor, a component of mTORC1, suggesting that mTORC2 has the predominant role in autophagy mediated by Pol $\gamma$ deficiency. To demonstrate mTORC2 activity, we employed an in vitro kinase assay using purified recombinant human AKT as the substrate. mTORC2, which was immunopurified using a Rictor affinity column, was able to phosphorylate AKT (S473), and the levels of phosphorylation of AKT were increased in Pol $\gamma$-deficient cells as compared to control cells (Fig. 5c). Similarly, to measure mTORC1, we performed an in vitro kinase assay using purified recombinant $\mathrm{S} 6 \mathrm{~K}$ as the substrate. mTORC1 was immunoprecipitated with Raptor antibody followed by addition of S6K. As shown in Fig. 5d, the phosphorylated p70S6K did not change in Pol $\gamma$-deficient cells as compared to the corresponding control cells. Immunopurified Rictor or Raptor from affinity column was confirmed by western blotting with Rictor and Raptor antibody, respectively (Fig. 5c, panel a, 
Fig. 3 Suppression of Pol $\gamma$ increases the autophagic response. a LC3 punctation was detected in Pol $\gamma$-deficient PCRISPR cells following expression of GFP-LC3. For each cell type, 100 GFP-positive cells were counted, and the right panel shows the quantification of punctated cells. b Western blot analysis and quantification of the increase in LC3 II, beclin 1, phosphorylated beclin 1, ATG7, and the decrease in p62 in Pol $\gamma$ deficient PCRISPR cells compared to controls. c Autophagy flux was detected by western blotting in PCRISPR cells following treatment with autophagy inhibitors (MnP; MnTnBuOE-2-PyP ${ }^{5+}$ 3-MA; 3methyle adenine and

Bafilomycin). The bar graph shows the quantification of LC3 II band intensity normalized to $\beta$-actin. d LC3 punctation was detected using fluorescence microscopy with or without autophagy inhibitors. The bar graph shows the quantification of punctated cells (100 GFPpositive cells were counted for each cell type). In all panels, each experiment was repeated at least three times. In the bar graphs, each data point represents the mean $\pm \mathrm{SD}$ of three individual samples. Statistical analysis was performed using $t$ tests for two groups or one-way ANOVA analysis and Bonferroni's posttest for multiple-group comparisons. Statistical significance is indicated by asterisks: $* p<0.05$ and $* * p<$ 0.01
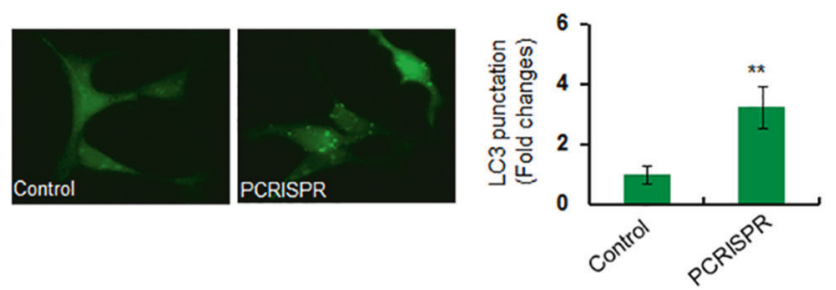

b

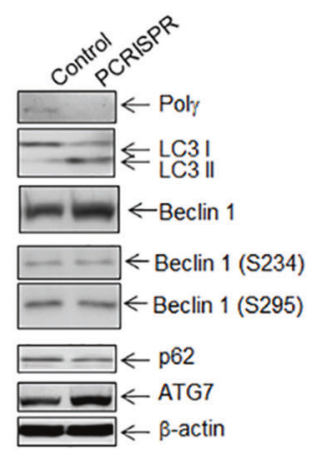

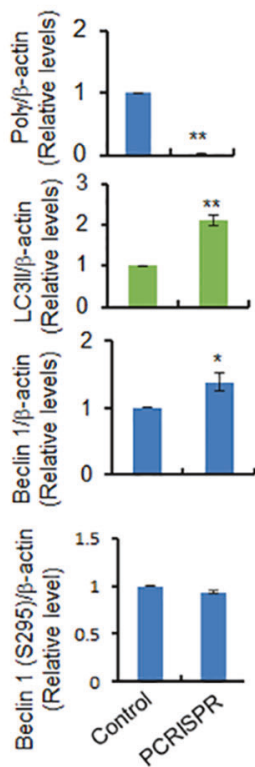

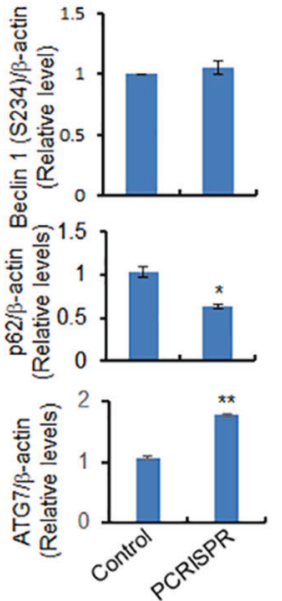

C

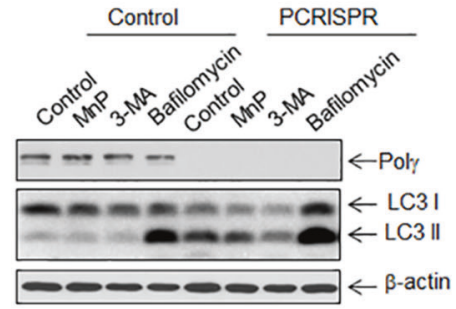

d

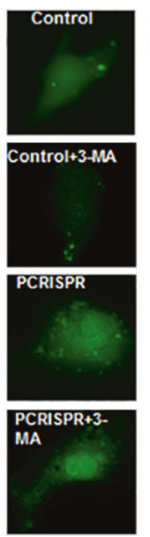

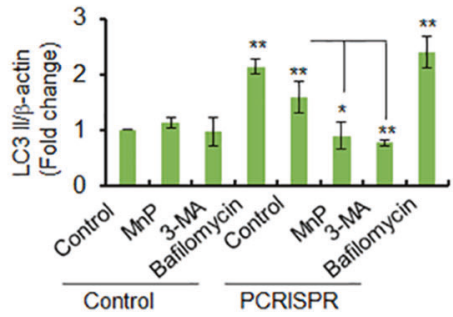

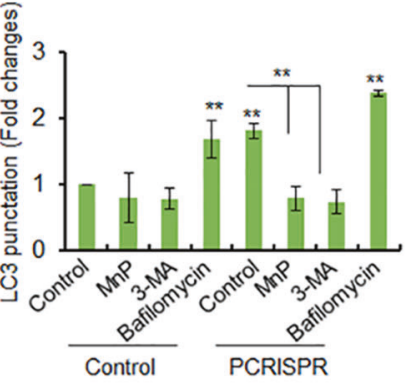

d, panel a). Equal loading of substrate was assured by detecting substrate levels using immunoblotting with AKT or p70S6K antibody, respectively (Fig. 5c, d). These results demonstrate the selective increase of mTORC2 in Pol $\gamma$-deficient cells. Next, we investigated mTOR complex formation in stable Pol $\gamma$-knockdown cells using 
a
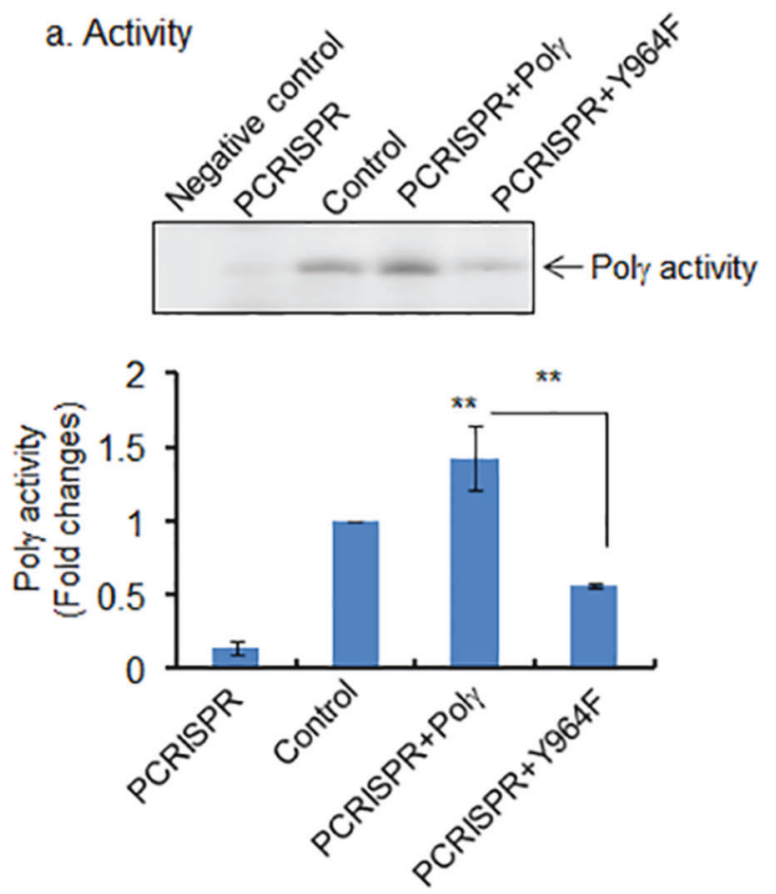

b. Western blot

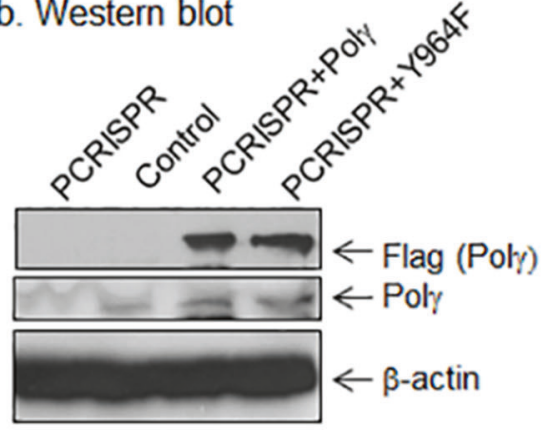

b

a. Activity
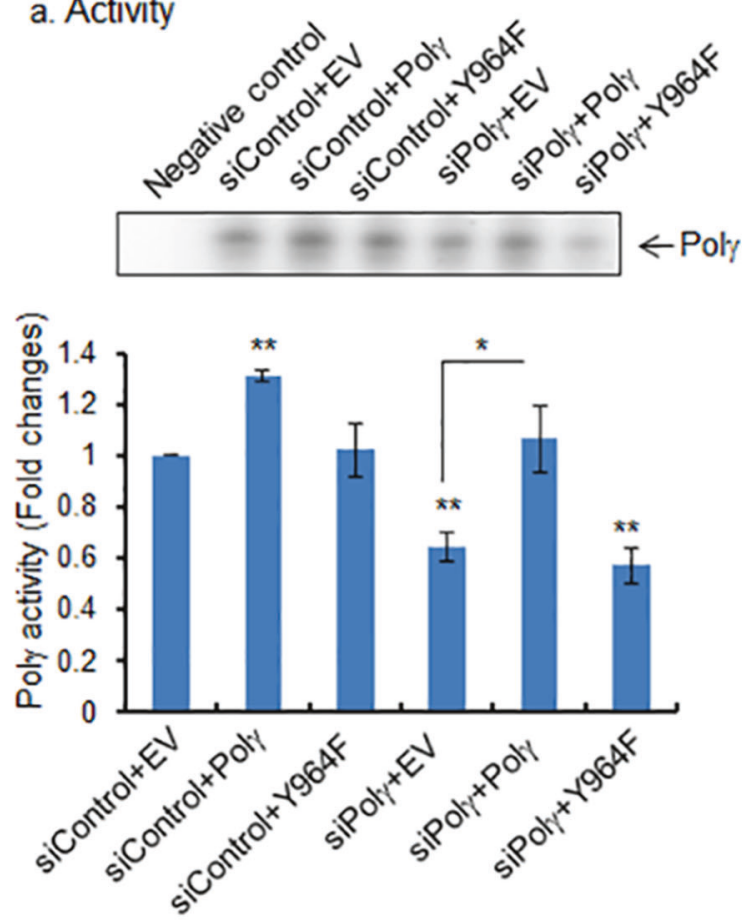

b. Western blot
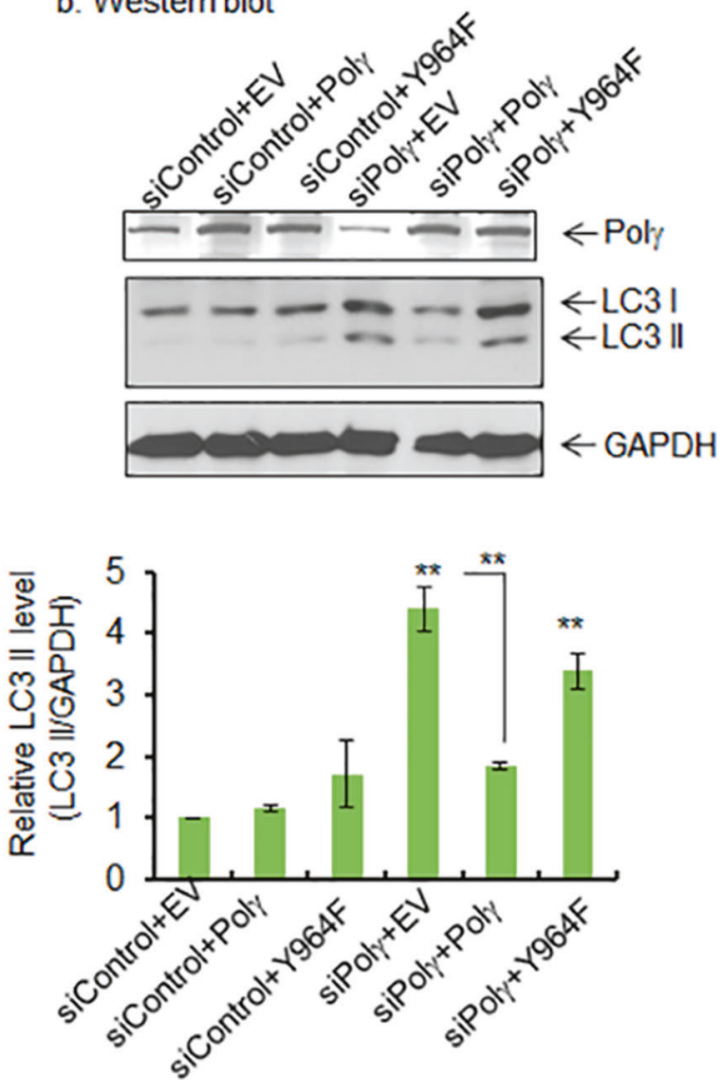

Rictor pulled down more mTOR in Polys, suggesting the preferential formation of $\mathrm{mTORC} 2$ following Pol $\gamma$ suppression (Fig. 5f). show that mTOR pulled down more Rictor than Raptor (Fig. 5e). Reverse co-immunoprecipitation data show that 
Fig. 4 Effects of tyrosine mutation on Pol $\gamma$ activity and autophagy. a, panel a The activity of Pol $\gamma$ in mitochondria following transfection of wild-type and mutant (Y964F) Pol $\gamma$ expression vectors in Pol $\gamma$-deficient PCRISPR cells. Relative activity of Pol $\gamma$ was measured and expressed as the fold change (bottom panel). The specificity of Pol $\gamma$ activity was validated by comparing with negative control, which contains template-primer mixture and radio-labeled ATP without enzyme. Panel $b$ The ectopic expression of wild-type and mutant Pol $\gamma$ was detected by western blotting using Flag antibody. Endogenous level of Pol $\gamma$ in PCRISPR cells is also shown. b, panel a The Pol $\gamma$ activity in JB6 cells following coexpression of wild-type or mutant (Y964F) Pol $\gamma$ plasmid along with Pol $\gamma$ siRNA. Relative activity of Pol $\gamma$ was measured and expressed as the fold change (bottom panel). Panel $b$ The suppression of Pol $\gamma$ by siRNA and the overexpression of wild-type and mutant Pol $\gamma$ were confirmed by western blotting using Pol $\gamma$ antibody. The relative level of LC3 II formation in JB6 cells following coexpression of wild-type or mutant (Y964F) Pol $\gamma$ plasmid along with Poly siRNA or control siRNA is shown (bottom panel). The specificity of Pol $\gamma$ activity was validated by comparing with negative control, which contains template-primer mixture and radiolabeled ATP without enzyme. Each data point represents the mean \pm SD of three individual samples. Statistical analysis was performed using one-way ANOVA analysis and Bonferroni's post-test for multiple-group comparisons. Statistical significance is indicated by asterisks: $* p<0.05$ and $* * p<0.01$

To address whether mTORC 2 that is preferentially activated in Pol $\gamma$-deficient cells is sensitive to an increase in ROS, we used ROS scavenger manganese superoxide dismutase mimetics $(\mathrm{MnP})$ and then coimmunoprecipitated with mTOR antibody. The results (Fig. 5g) show that more Rictor was pulled down with mTOR antibody in Pol $\gamma$ deficient cells as compared to controls. Treatment of Polydeficient cells with MnP significantly reduced the Rictor levels in the immunocomplex, suggesting that mTOR/Rictor complex formation is ROS-dependent. Consistently, reverse immunoprecipitation with Rictor antibody in Pol $\gamma$ knockdown cells showed a decrease of mTOR-Rictor interaction after MnP treatment (Fig. 5g). In addition, MnP treatment decreased the Rictor protein level and AKT phosphorylation suggesting that oxidative stress induces AKT activation (Figure S3B). Further, inhibition of AKT phosphorylation was achieved by treating the cells with the AKT inhibitor as well as by siRNA silencing, which significantly suppressed the Pol $\gamma$-deficiency-mediated LC3 II levels, suggesting that the AKT pathway is associated with oxidative stress-induced survival autophagy (Supplementary Figure S3A, S3C).

\section{Polp deficiency-mediated autophagy is Rictor- dependent}

The role of mTORC2 in autophagy is not well understood. We thus tested the role of Rictor vs. Raptor in autophagy by employing Rictor suppression or overexpression. We first confirmed by western blotting that the proteins were ectopically expressed (Fig. 6a). Our data show that the basal level of LC3 punctation is significantly increased following overexpression of Rictor but not of Raptor in mouse skin cells (Fig. 6b). To further demonstrate the role of Rictor in Pol $\gamma$ deficiency-mediated autophagy, we suppressed Poly using siRNA in stable Rictor-knockout MEF cells. Rictorknockout reversed the increase in LC3 punctation observed in MEF cells with overexpression of Pol $\gamma$ siRNA (Fig. 6c). In addition, as shown in Fig. 6d, Rictor-knockout cells have decreased levels of LC3 II, beclin 1, and ATG7, but not p62, as compared to control MEF cells. Suppression of Poly by siRNA in MEF cells resulted in increased LC3 II, beclin 1, and ATG7 expression. By contrast, suppression of Poly by siRNA in Rictor-knockout MEF cells did not increase LC3 II, beclin 1, or ATG7 expression (Fig. 6d). These results suggest that Rictor plays an important role in Poly deficiency-mediated autophagy.

To further demonstrate the role of Rictor, we modulated Rictor levels by overexpression. As shown in Fig. 6e, we observed an increase in LC3 cleavage to LC3 II following overexpression of Rictor but not Raptor in Rictor-knockout MEF cells. Consistently, AKT phosphorylation was increased in Rictor-knockout MEF cells following overexpression of Rictor but not Raptor. These results are consistent with the idea that Rictor plays a critical role in inducing autophagy in response to Poly deficiency.

\section{Deficiency of Poly enhances cell proliferation}

We then asked whether Pol $\gamma$ deficiency enhances cell proliferation, because autophagy can function in both cell survival and cell death pathways. We employed a gold standard soft-agar colony formation assay to assess the cell proliferation. Our data show that in treated cells vs. control cells, MnP, 3-MA, and bafilomycin had no effect on cell growth (Fig. 7a). Cell growth was significantly increased in PCRISPR cells as compared to controls (Fig. 7b), and the increased cell growth was suppressed by inhibitors of autophagy in Poly-deficient cells. The DNA synthesis-based cell proliferation assay using BrdU uptake was consistent with the increase in cell growth (Fig. 7c). These results suggest that a deficiency of Pol $\gamma$ increases cell proliferation.

To address whether Rictor has a direct role in cell proliferation, we investigated the effects of Rictor in the cell proliferation assay. The increase in phosphorylated p70S6K (Th389) was also inhibited in Rictor-deficient Pol $\gamma$-knockdown cells (Fig. 7d). Figure 7e shows that the increase in BrdU uptake in Pol $\gamma$-deficient cells was abolished in Rictordeficient cells. Consistently, BrdU uptake was increased in stable Pol $\gamma$-knockdown cells, and was significantly reduced again upon treatment with AKT inhibitor (Fig. 7f). This result provides mechanistic evidence that Pol $\gamma$ deficiencymediated autophagy increases cell proliferation via AKT activation. 
a

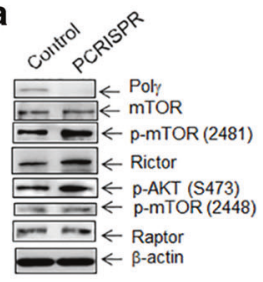

.

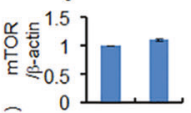

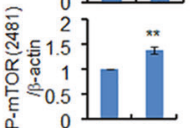

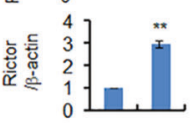

然

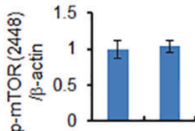

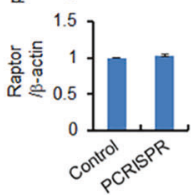

e

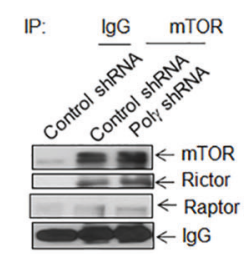

Input
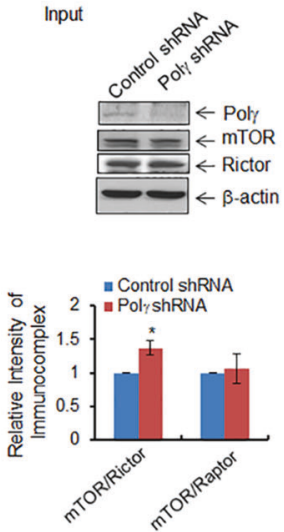

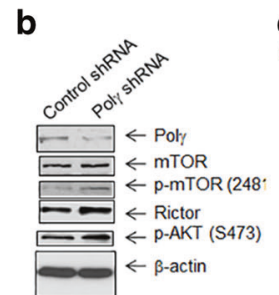

C

a. Rictor level in IP pellet

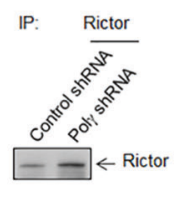

Input
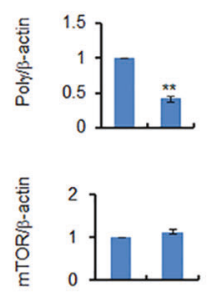

然
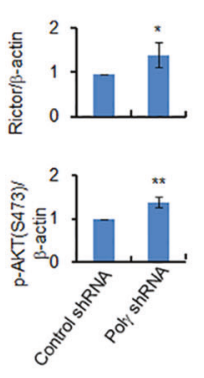

b. mTORC2 kinase activity

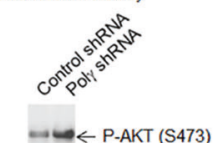

$\leftarrow \lg G$

$=$ Loaded AKT substrate

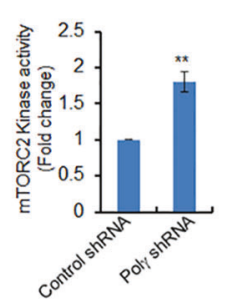

d ${ }_{\text {a. Raptor level in IP pellet }}$

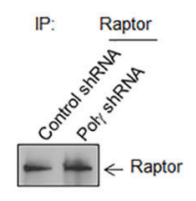

Input

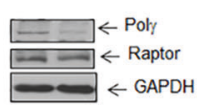

b. mTORC1 kinase activity

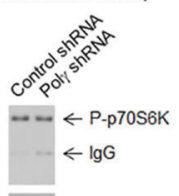

$\leftarrow$ Loaded AKT substrate

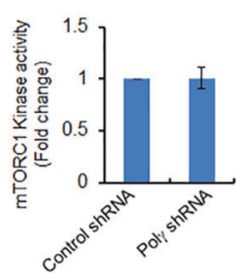

g

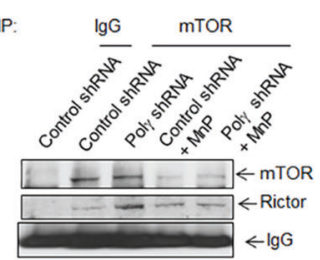

Input
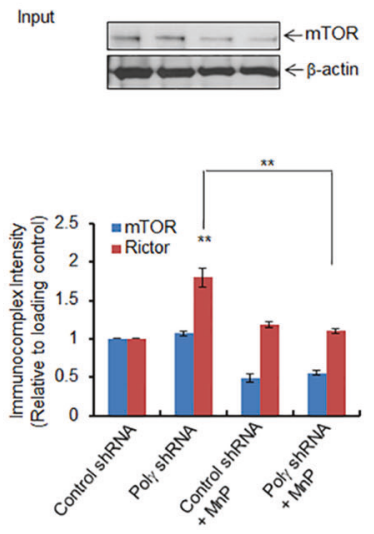

Input
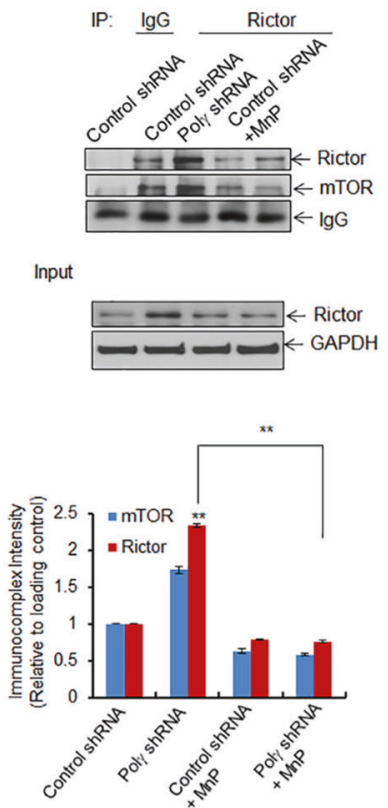
4 Fig. 5 Activation of AKT/mTOR pathway following Pol $\gamma$ deficiency. a, b Increased levels of mTOR phosphorylation at S2481, AKT phosphorylation at S473, and Rictor were detected by western blotting in a Pol $\gamma$-deficient PCRISPR cells and $\mathbf{b}$ cells stably expressing Pol $\gamma$ shRNA. c, $\mathbf{d}$ In vitro mTOR kinase activities were measured by using specific substrates for mTOR complexes coupled with immunoprecipitation and western blotting in cells stably expressing Pol $\gamma$ shRNA. c For mTORC2 kinase activity, total cell lysates were immunoprecipitated with Rictor antibody. Panel a The immunoprecipitated proteins were detected by western blotting using Rictor antibody. Panel b Immunoprecipitated product was then incubated with purified AKT. The phosphorylated AKT (S473) was detected as an indicator of mTORC2 kinase activity. d, Panel a The immunoprecipitated proteins were detected by western blotting using Raptor antibody. For mTORC1 kinase activity, total cell lysates were immunoprecipitated with raptor antibody. Immunoprecipitated product was then incubated with purified p70S6K protein and the phosphorylated p70S6K was detected by western blotting as an indicator of mTORC1 activity. e Coimmunoprecipitation of Rictor and Raptor with mTOR from the total cell lysates of cells expressing Pol $\gamma$ shRNA. Input controls are provided as loading control. f Reverse coimmunoprecipitation with Rictor antibody followed by western blotting performed with Rictor, mTOR, and Raptor antibodies. Input controls were provided as loading control. g Immunoprecipitation was also carried out using mTOR antibody in JB6 cells stably expressing Poly shRNA following pretreatment with $\mathrm{MnP}(20 \mu \mathrm{M} \times 24 \mathrm{~h})$. The immunoprecipitated products were analyzed by western blotting using antibodies against mTOR and Rictor. Input controls were added as loading control. Similarly, reverse immunoprecipitation was performed using Rictor antibody in Pol $\gamma$ deficient cells following pretreatment with MnP. Input controls are also shown as loading control. All immunoprecipitated proteins were normalized with house-keeping loading control and quantified. Each experiment was repeated at least three times. In the bar graphs, each data point represents the mean \pm SD of three individual samples. Statistical analysis was performed using $t$ tests for two groups or one-way ANOVA analysis and Bonferroni's post-test for multiple-group comparisons. Statistical significance is indicated by asterisks: $* p<0.05$ and $* * p<0.01$

\section{Discussion}

In this study, we demonstrate for the first time that oxidative modification of Pol $\gamma$ and subsequent inactivation of Pol $\gamma$ selectively trigger prosurvival autophagy responses via Rictor-mediated mTORC2. mTOR is a key regulator of autophagy and cell growth [27]. Although the role of mTORC1 is well documented, the role of mTORC2 remains unclear. Here, we demonstrated that perturbation of mitochondrial energy metabolism due to deficiency of Poly causes an increase in ROS, which activates Rictor to initiate prosurvival autophagy. Our findings support that activation of mTORC2 is a mitochondria-mediated event, at least under the conditions of mitochondrial oxidative stress due to Pol $\gamma$ deficiency.

It has been shown that nitric oxide and superoxide radical generated by UVB radiation can form peroxynitrite, leading to nitration of proteins $[17,18]$. Specific tyrosine nitration and inactivation of enzymatic activity by peroxynitrite have been reported for MnSOD and aconitase [28,
29]. Our data indicate that Pol $\gamma$ is nitrated when human and mouse keratinocytes are exposed to UVB radiation, confirming our previous data for mouse skin tissues [9]. Importantly, we identified Y964 in the active site of DNA Pol $\gamma$ as the primary site of peroxynitrite-induced inactivation of Poly (Fig. 1). Furthermore, tyrosine mutation to phenylalanine at position 964 of mouse Pol $\gamma$ suppressed the enzymatic activity, supporting the relevance of tyrosine nitration and loss of enzymatic function. Consistent with the function of Poly in mtDNA repair, we observed significant mtDNA damage and impairment of mitochondrial function in Pol $\gamma$-deficient cells that led to decreases in ATP-linked oxygen consumption, increased glycolysis, and higher ROS levels. We also have demonstrated that Poly deficiency increases autophagy markers such as LC3 11 formation, beclin 1, and ATG7, as well as activates ribosomal kinase. These results are consistent with the concept that Poly deficiency initiates signaling in prosurvival autophagy pathways.

The mechanism by which Pol $\gamma$ deficiency enhances prosurvival autophagy is unclear. Our data suggest that Pol $\gamma$ deficiency led to metabolic reprogramming in Pol $\gamma$-deficient cells, which then generated ROS to activate mTOR pathways. It has been reported that the activation of mTOR is required in immune cells [30] during proliferation. Furthermore, endotoxin-induced autophagy is a survival mechanism that drives proliferation of hepatocytes [31] and in cardiac tissues [32]. The central components of the cell survival pathway, including AKT and mTOR, sense cellular metabolic alterations and trigger cell survival [24]. Our results demonstrate an increase in the phosphorylation of mTOR and AKT in Poly-deficient cells (Fig. 5), supporting the previous findings that AKT-mTOR regulates autophagy and cell survival. Although it has been demonstrated that the PI3K-AKT-mTOR pathway mediates antiautophagic signaling, inhibition of mTOR by rapamycin induces autophagic cell death [33], and recent studies have shown that autophagy is also induced through activation of AKTmTOR pathways [34, 35]. In yeast, the TOR complex-1, which is similar to the mTOR regulatory protein raptor that is a key regulator of translation and ribosome biogenesis, is responsible for the induction of autophagy [36]. Recently, it has been reported that AKT increases autophagy upon activation (by phosphorylation at S473) and consequently forms a complex with lysosomal proteins [37]. Serinethreonine kinase AKT and mTOR are well connected in their signaling pathway, whereas mTORC2 is known to phosphorylate AKT at S473. Our data show an increase in AKT phosphorylation at S473 as well as a preferential formation of mTORC 2 over mTORC1 in Pol $\gamma$-deficient cells (Fig. 5). Further, our strategy of overexpression and knockdown of Rictor, which is an important component of mTORC2, clearly demonstrates the role of Rictor in 
a

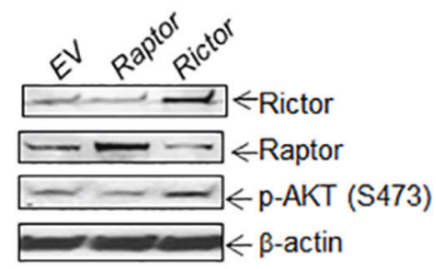

b
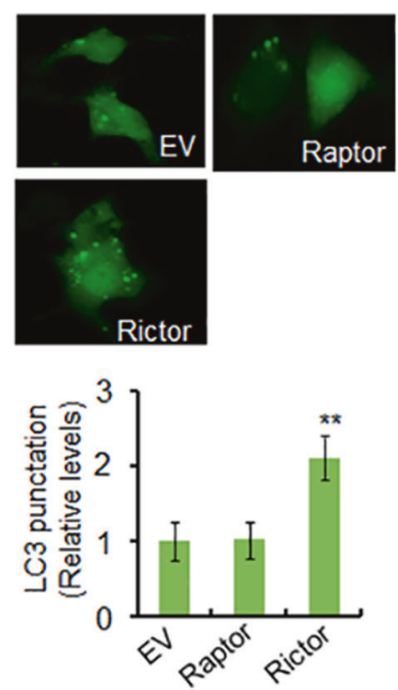

c

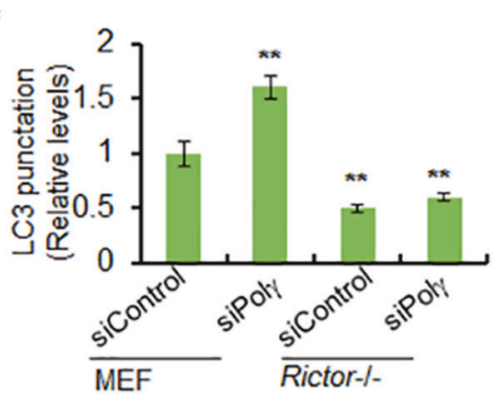

d
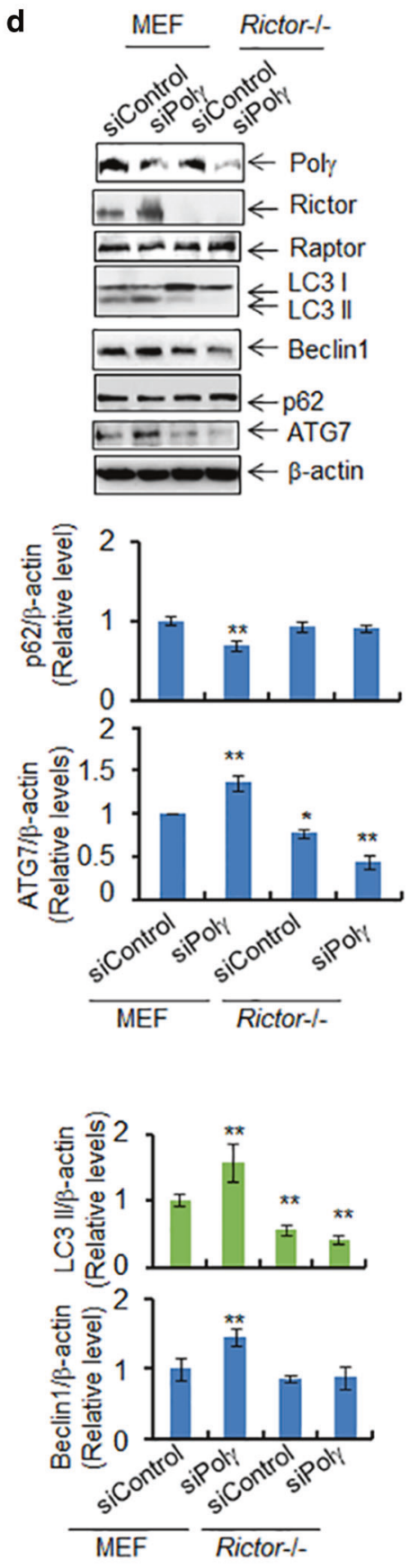

e

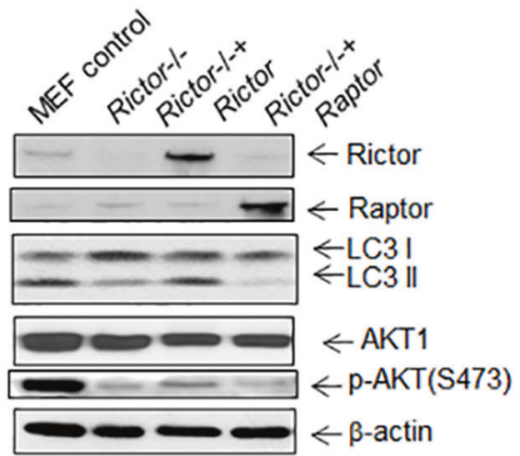

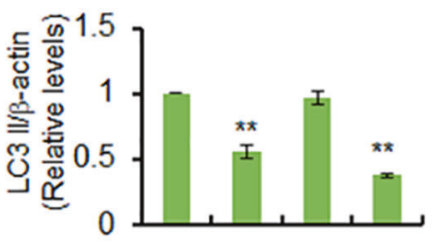
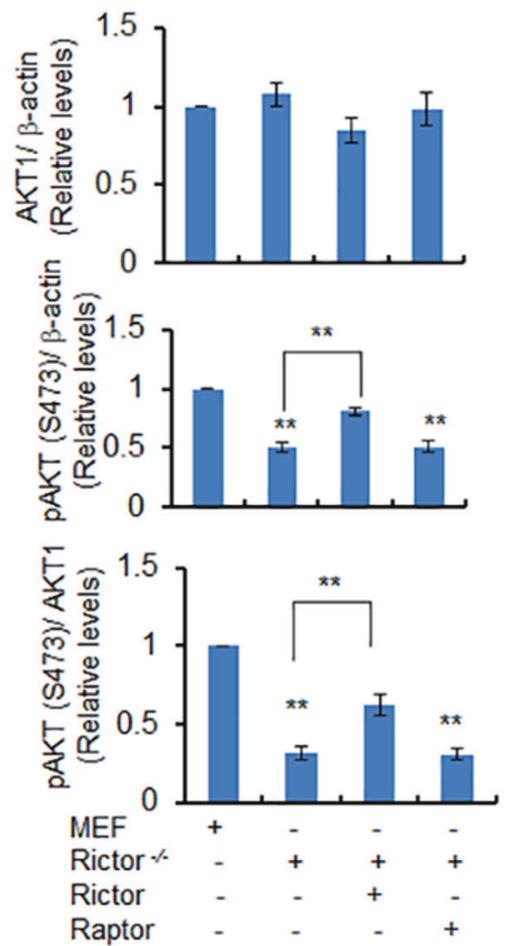

Fig. 6 Pol $\gamma$ deficiency-mediated autophagy is RICTOR-dependent. a Western blotting shows the overexpression of Rictor or Raptor following transfection of the corresponding vectors in JB6 cells. AKT phosphorylation at Ser473 in the presence of the overexpressed Rictor was also detected by western blotting. Plasmid vector without any protein expression sequence was used as control and is designated by EV. b Detection of LC3 punctation following overexpression of Rictor or Raptor. Quantification of LC3 punctation in Raptor- and Rictoroverexpressing JB6 cells is shown (bottom). c Quantification of LC3 punctation in wild-type MEF and Rictor-knockout MEF cells following Pol $\gamma$ siRNA transfection. d Effects of Pol $\gamma$ deficiency in MEF and Rictor-knockout cells are achieved by expression of Poly siRNA. The endogenous autophagy markers (LC3 II, beclin 1, ATG7, and p62) were detected by western blotting. The right panels show the corresponding quantification. e Overexpression of Rictor or Raptor in Rictor-knockout MEF cells and the level of LC3 II were determined by western blotting. AKT phosphorylation at Ser473 induced by Rictor overexpression was also detected by western blotting. In all panels, each experiment was repeated at least three times, and statistical analysis was performed using one-way ANOVA analysis followed by Bonferroni's post-test for multiple-group comparisons. Statistical significance is indicated by asterisks: $* p<0.05$ and $* * p<0.01$ 
a

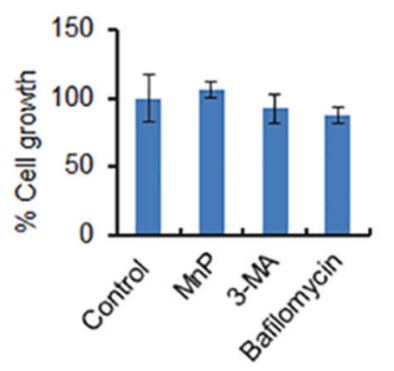

b

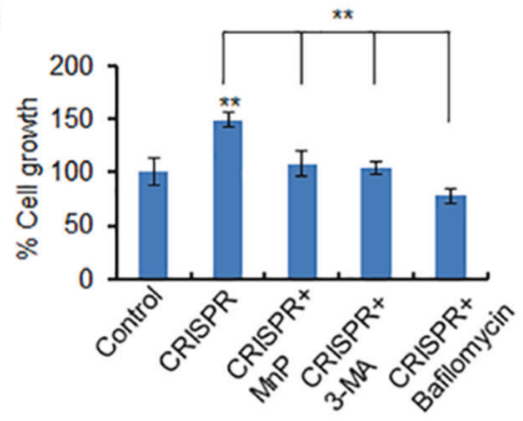

C

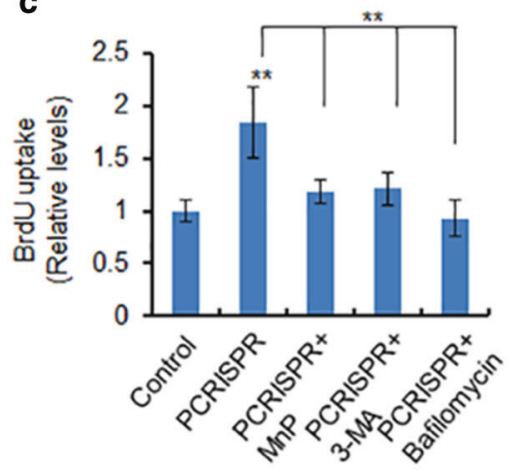

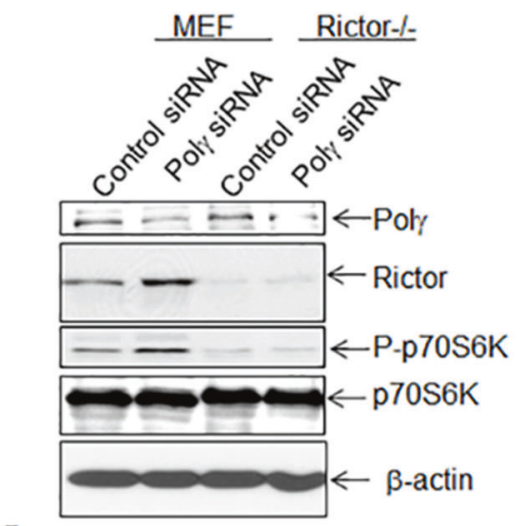
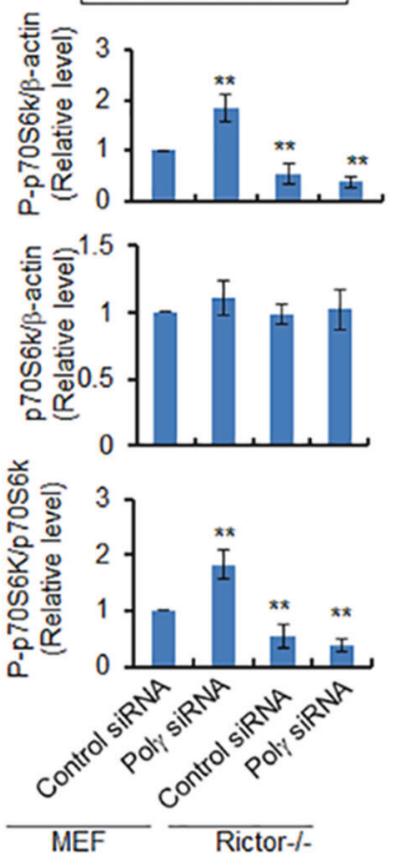

e

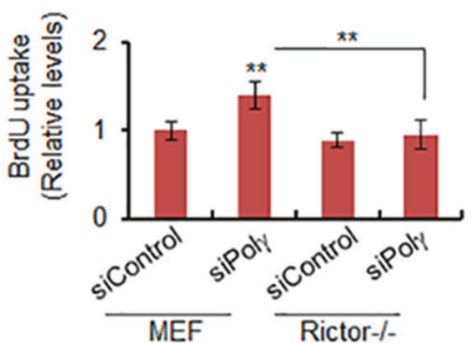

f

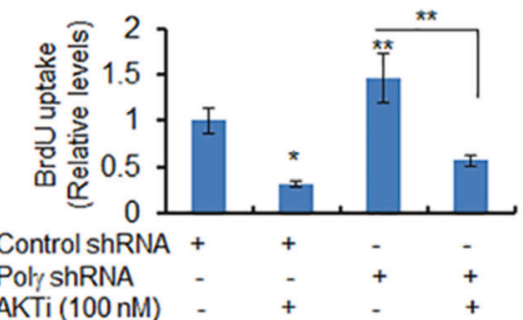

Fig. 7 Deficiency of Pol $\gamma$ enhances autophagy-dependent cell proliferation. a The cytotoxicity of ROS and autophagy inhibitors (MnP, $20 \mu \mathrm{M}$; 3-MA, $2.5 \mathrm{mM}$; and Bafilomycin, $50 \mathrm{nM}$ ) was assessed in JB6 cells $24 \mathrm{~h}$ after treatment by determining cell growth. b Cell growth was also measured in PCRISPR cells following treatment with the indicated inhibitor. $\mathbf{c}$ BrdU uptake was determined as a measure of cell proliferation in PCRISPR cells with or without ROS or autophagy inhibitors. d Phosphorylation of p70S6K was detected by western blotting in wild-type and Rictor-knockout MEF cells following suppression of Pol $\gamma$ by siRNA. e Cell proliferation was determined in
Rictor-knockout cells after Poly suppression using the BrdU uptake assay (assay details are given in Materials and Methods section). f Cell proliferation was also determined in Pol $\gamma$-knockdown cells following treatment with AKT inhibitor using the BrdU uptake assay. In all panels, each data point represents the mean \pm SD of three individual samples. Statistical analysis was performed using one-way ANOVA analysis and Bonferroni's post-test for multiple-group comparisons. Statistical significance is indicated by asterisks: $* p<0.05$ and ${ }^{* *} p<$ 0.01

nitration of Pol $\gamma$ and subsequent catalytic inactivation of Pol $\gamma$ trigger prosurvival autophagy responses selectively via the Rictor-mediated mTORC2, which is schematically depicted in Fig. 8. These results provide critical insights into the mechanism of mitochondrial dysfunction-mediated autophagy that have broad implications for our understanding of physiological and pathological conditions leading to the development of cancer and other metabolismrelated diseases. 


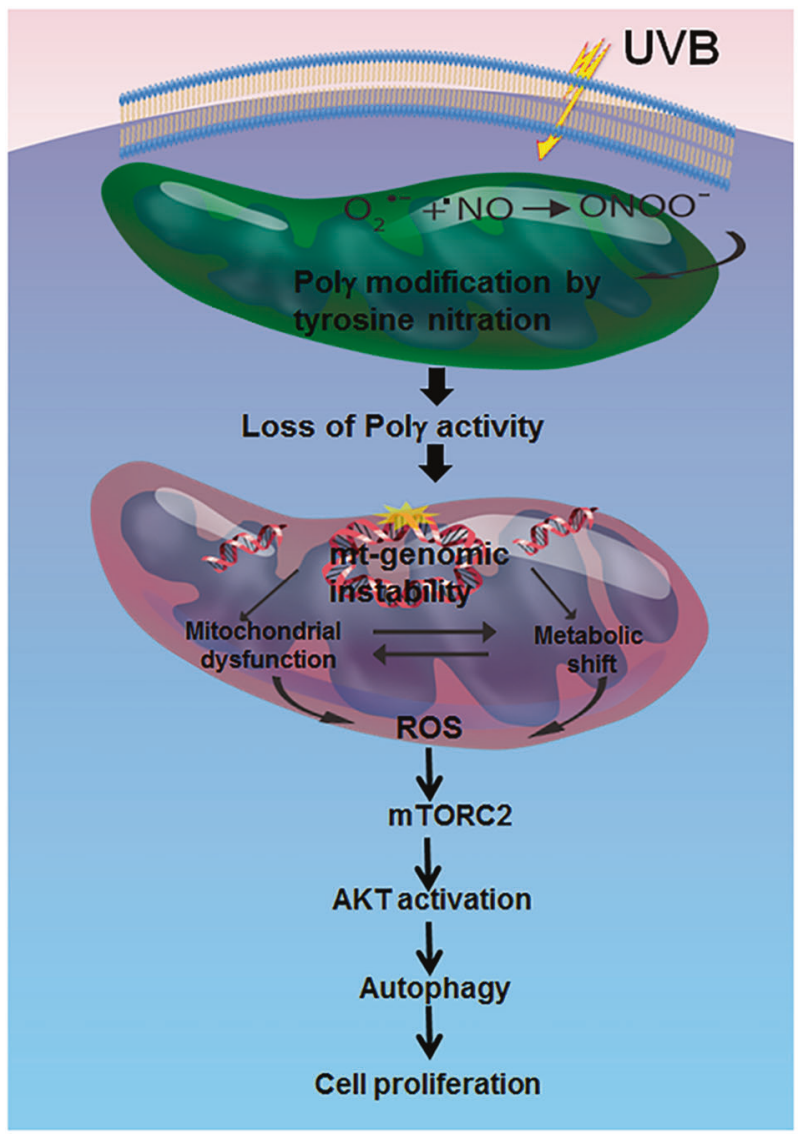

Fig. 8 Summary of the proposed role of Pol $\gamma$ in prosurvival autophagy. Schematic diagram of the impact of Poly deficiency and subsequent loss of enzymatic activity on the mTORC2/AKT pathway during prosurvival autophagy

\section{Materials and methods}

\section{Reagents}

Anti-LC3A/B (Cat. 1274), anti-Beclin 1 (Cat. 3495), and ATG7 (Cat. 8558) were purchased from Cell Signaling Technology (Danvers, MA). Both anti- $\beta$-actin (Cat. SAB2100037), anti-mTOR (Cat. PLA0114), and anti-Flag (F3165) antibodies were purchased from Sigma (St. Louis, MO). The rabbit polyclonal Poly (Cat. PA5-75881) was purchased from Thermo Scientific (Waltham, MA). AntiRaptor (Cat. 05-1470) and anti-Rictor (Cat. 05-1471) antibodies were purchased from Millipore (Temecula, CA). Poly siRNA (Cat. Sc-155884) and the lentiviral Pol $\gamma$ shRNA plasmid (Sc-155884-SH) were purchased from Santa Cruz Biotechnology (Santa Cruz, CA). PCR amplified full-length Pol $\gamma$ cDNA was sucloned into pEGFP-N1Flag expression vector (Addgene, Cat. 60360), and the pEGFP-LC3 plasmid was a kind gift of Dr. Xianglin Shi, University of Kentucky (Lexington, KY). MnTnBuOE-2$\mathrm{PyP}^{5+}(\mathrm{MnP})$ was the kind gift of Dr. Ines Batinic-Haberle,
Duke University School of Medicine (Durham, NC). 3Methyle adenine (3-MA, Cat. tlrl-3ma) and Bafilomycin A1 (Cat. tlrl-baf1) were purchased from InvivoGen (San Diego, $\mathrm{CA})$. 10-nonylacridine orange bromide (NAO) was purchased from Thermo Fisher (Cat. A1372) All other chemicals were purchased from Sigma unless otherwise specified.

\section{Cell culture and treatments}

The JB6 mouse skin epidermal cell line was originally obtained from Dr. Nancy H. Colburn of the National Cancer Institute, MD, and was maintained as described previously [40, 41]. The primary human epidermal keratinocyte (HEKn, Cat. C-001-5C) cells were purchased from Invitrogen Life Sciences (Carlsbad, CA). Both wild-type and Rictor-knockout MEFs were originally obtained from David Sabatini (Whitehead Institute, MA). All cells were grown in a $5 \% \mathrm{CO}_{2}$ incubator at $37^{\circ} \mathrm{C}$ in media consisting of either MEM supplemented with $10 \%$ fetal bovine serum (Hyclone Inc., Logan, UT), 1\% (w/v) L-glutamine (Invitrogen), and $1 \% \mathrm{P} / \mathrm{S}$ antibiotics (Invitrogen) or Epilife medium supplemented with S7 (Invitrogen) for primary cells. Cells were exposed to a single dose of UV radiation $\left(50 \mathrm{~mJ} / \mathrm{cm}^{2} \times 1 \mathrm{~h}\right)$ using UVB lamps as described previously [9]. Cells were also treated with $20 \mu \mathrm{M} \mathrm{MnP}, 2.5 \mathrm{mM}$ 3-MA, and $50 \mathrm{nM}$ Bafilomycin A1 for $24 \mathrm{~h}$.

\section{Transfection}

Cells were grown for $24 \mathrm{~h}$ with no antibiotics to obtain 70-80\% confluency. The cells were then transfected with plasmids following a transfection protocol using Lipofectamine ${ }^{\circledR}$ as directed by the manufacturer. Cells were transfected with $1-2 \mu \mathrm{g}$ of plasmid DNA containing either Poly or LC3-GFP (equilibrated to the same amount of DNA by adding control vector) or control vector alone. Twentyfour hours after transfection, the cells were washed twice with phosphate-buffered saline and incubated in fresh medium for another $24 \mathrm{~h}$ with or without treatment for an indicated time. Cells were then processed for whole cell lysate preparation or fluorescence microscopy. Similarly, siRNAs were transfected using Transfectin ${ }^{\oplus}$ (Santa Cruz Biotechnology) according to the manufacturer's protocol. Cells were exposed to the siRNA for $72 \mathrm{~h}$. The siRNA sequences targeting Pol $\gamma$ are presented in Supplementary Table S1.

\section{Poly shRNA stable clones}

Lentiviral constructs containing mouse Pol $\gamma$ shRNA (Santa Cruz Biotechnology) were used to make JB6 cells with stable knockdown of Pol $\gamma$ protein. Lentiviral constructs 
expressing an empty vector were used as controls. The lentiviral constructs were packaged using the manufacturerprovided trans-lentiviral packaging system before transduction into JB6 cells in accordance with the manufacturer's protocol. For stably expressing Poly shRNA, JB6 cells were selected using puromycin. Stable expression of Poly in pools of cells was verified by western blotting using Poly antibody.

\section{Deletion of the Poly gene in JB6 cells by CRISPR}

The D10A Cas9n CRISPR system [42] was used to introduce two DNA single-strand breaks to minimize off-target effects. The genome sequences containing the guide/pam sequences in the mouse Poly gene are: (A) 5'-CCT/TCGTGGTAACC CTGTCCACT- $3^{\prime}$ (reverse complementary) and (B) 5'TTGCCTCTTTCAGGTATTCC/TGG- $3^{\prime}$. The two sequences are adjacent, and thus by combination Cas9n introduces a DNA double-strand break in the beginning of exon 3 (for details, see Fig. 2 legend). To construct a Cas9n vector targeting site (A), a linker was prepared by annealing $5^{\prime}$-CACC GAGTGGACAGGGTTACCACGA-3' and 5'-AAACTCGT GGTAACCCTGTCCACTC-3', followed by phosphorylation of the $5^{\prime}$-ends by $\mathrm{T} 4$ polynucleotide kinase. The linker was then cloned into the pSpCas9n(BB)-AA-Puro vector (Addgene, Cambridge, MA; Cat. 62988) that had been prepared by cutting with BbsI. Similarly for (B), a linker (5'CCGTTGCCTCTTTCAGGTATTCC-3' annealed with 5'AACGGAATACCTGAAAGAGGCAA-3') was cloned into pD1411-AP (DNA2.0 Inc., Newark, CA) that had been cut with SapI. Deletion in the locus was confirmed by PCR of the genomic DNA using primers 5'-AAGACACAAGGGGTTG GTCC-3' and 5'-AGACGGTGTTGTCAAAGTTTGT-3', and then the absence of Poly protein expression was confirmed by immunoblotting.

\section{In vitro kinase assay for mTORC1 and mTORC2 activity}

The in vitro kinase assays for mTORC1 and mTORC2 activity were performed according to the protocol adopted from Dr. David Sabatini's laboratory. Briefly, cells were lysed in $200 \mu \mathrm{L}$ of lysis buffer containing $40 \mathrm{~mm}$ HEPES, $\mathrm{pH} 7.5,120 \mathrm{mM} \mathrm{NaCl}, 0.3 \%$ CHAPS, $1 \mathrm{mM}$ EDTA, $2.5 \mathrm{mM}$ sodium pyrophosphate. Cell lysate was passed through a sepharose-affinity column conjugated with antiraptor or anti-Rictor antibody (Cell Signaling, CA). After appropriate washing with a kinase buffer containing $1 \mathrm{mM}$ dithiothreitol, beads were incubated for $30 \mathrm{~min}$ at $30^{\circ} \mathrm{C}$ with purified recombinant $\mathrm{S} 6 \mathrm{~K}$ and $\mathrm{AKT}$ as substrate for mTORC1 and mTORC2 activity, respectively. The reactions were then terminated by boiling in the presence of $1 x$ SDS sample buffer. Phosphorylation was detected by western blotting using anti-pp70S6K antibody for mTORC1 activity and antiphosphorylated AKT (S473) antibody for mTORC2 activity.

\section{Site-directed mutagenesis}

Specific point mutation of Pol $\gamma$ was performed using the QuickChange Site-Directed Mutagenesis kit (Agilent Technologies, Santa Clara, CA; Cat. 200524) as described previously [43]. Primer sets were designed to mutate tyrosine (Y964) to phenylalanine in mouse Poly using the primers (a) 5'-AGGCCTGCGCCGGCCTCGGCTGTCTG CG-3' (forward) and

(b) 5'-CGCAGACAGCCGACCGGCGCAGGCCT-3' (reverse). The clonal DNAs were propagated in E. coli. Clones carrying a single mutation were confirmed by DNA sequencing.

\section{Poly reverse transcriptase activity assay for pure protein}

The RNA-dependent DNA polymerase activity of purified Pol $\gamma$ was measured as described previously [44]. Briefly, a $50 \mu \mathrm{L}$ reaction mixture with $10 \mu \mathrm{g}$ of the mitochondrial protein in $25 \mathrm{mM}$ HEPES-KOH $\mathrm{pH} 8.0,0.5 \mathrm{mM} \mathrm{MnCl}_{2}$, $100 \mathrm{mM} \mathrm{NaCl}$, and $2.5 \mathrm{mM} \beta$-mercaptoethanol; $50 \mu \mathrm{g} / \mathrm{mL}$ poly(rA); oligo(dT) $)_{12-18} ; \quad 100 \mu \mathrm{g} / \mathrm{mL}$ acetylated bovine serum albumin (BSA); $0.1 \mathrm{mM}$ aphidicolin; $500 \mu \mathrm{g} / \mathrm{mL}$ RNasin ${ }^{\circ}$ Rase inhibitor; and $5 \mu \mathrm{M}\left[\alpha-{ }^{32} \mathrm{P}\right]$ thymidine $5^{\prime}$ triphosphate was incubated at $37^{\circ} \mathrm{C}$ for $10 \mathrm{~min}$. The reaction was stopped with $1.0 \mathrm{~mL}$ of stop solution $(500 \mathrm{mM}$ $\mathrm{NaOH}, 100 \mathrm{mM}$ sodium pyrophosphate, $0.1 \mathrm{mg} / \mathrm{mL}$ calf thymus DNA, $0.5 \mathrm{mg} / \mathrm{mL}$ BSA). The DNA was precipitated with $20 \%$ trichloroacetic acid and radioactivity was measured by a liquid scintillation counter.

\section{Poly activity assay in isolated mitochondria}

Pol $\gamma$ activity in mitochondria was measured according to the method described by Szczesny [45]. Briefly, the activity of Pol $\gamma$ was measured using primer (5'-GACCCGATCTGATCCGATTCG-3') and template (5'-ATCCAACCTCG CGGTCGTATCGAATCGGATCAGATCGGGTCGTCA-

A- $\left.3^{\prime}\right)$. The primer-template was annealed and added to $20 \mu \mathrm{L}$ of reaction mixture containing $50 \mathrm{mM}$ Tris- $\mathrm{HCl}(\mathrm{pH}$ 8.6), $50 \mathrm{mM} \mathrm{KCl}, 2 \mathrm{mM} \mathrm{MgCl}, 20 \mu \mathrm{M}$ each of three unlabeled dNTPs, $2 \mu \mathrm{Ci}$ of $\left[\alpha^{-32} \mathrm{P}\right] \mathrm{dATP}$ and $3 \mu \mathrm{g}$ of the mitochondria. After incubation at $37^{\circ} \mathrm{C}$ for $30 \mathrm{~min}$, the reaction was terminated by the addition of $5 \mu \mathrm{L}$ of $70 \%$ formamide. The amplified DNA was resolved by $20 \%$ acrylamide/7 M urea gel electrophoresis. The radioactivity of the bands was quantified using Image Quanta software. 


\section{Liquid chromatography electrospray ionization- tandem mass spectrometry (LC-ESI-MS/MS) analysis}

The trypsin digestion and LC-MS/MS analysis of pure Poly were performed as reported previously $[42,46]$. The tryptic peptides were analyzed using an LTQ-Orbitrap mass spectrometer (Thermo Scientific, MA) coupled with an Eksigent Nanoflex cHiPLC ${ }^{\text {TM }}$ system (Eksigent, CA) through a nanoelectrospray ionization source. The peptides were separated with a reverse-phase cHiPLC at a flow rate of $300 \mathrm{~nL} / \mathrm{min}$. The LC-MS/MS data were submitted to a local mascot server for identification of peptide modifications via Proteome Discoverer (version 1.3) against the musculus taxonomy subset of the Swissprot database.

\section{Measurement of oxygen consumption rate and extracellular acidification rate}

XF extracellular flux assays (Seahorse-Bioscience, MA) were utilized to measure the oxygen consumption rate (OCR) and the extracellular acidification rate (ECAR). The OCR experiments were performed by sequentially adding the substrate oligomycin, carbonyl cyanide-4-(trifluoromethoxy) phenylhydrazone (FCCP), and antimycin/rotenone to cells and the ECAR measurements were performed by using glucose, oligomycin, and 2-deoxyglucose as substrate.

\section{Western blotting}

Proteins were analyzed by western blotting. Briefly, cell extracts were subjected to $10 \%$ or $12 \%$ SDSpolyacrylamide gel electrophoresis and transferred to a nitrocellulose membrane. Following blocking with 5\% BSA, membranes were then probed with specific primary antibody (by diluting at a range from 500 to 5000) followed by secondary antibody (dilution range 2000 to 6000) to detect specific proteins. Protein bands were detected using the enhanced chemiluminescence detection system (ECL ${ }^{\circ}$, Amersham Bioscience). Densitometric analysis was performed for quantification of proteins using ImageJ software (NIH).

\section{Fluorescence microscopy}

Green florescent LC3 puncta formation was detected by fluorescence microscope (model IX71, Tokyo, Japan) in live cells following transient transfection of pEGFP-LC3 expression vector.

\section{Immunoprecipitation}

Immunoprecipitation studies were carried out as described previously [43] with whole cell extracts using specific antibodies in a binding buffer (9.1 mM NaHPO4, $1.7 \mathrm{mM}$ $\mathrm{NaH}_{2} \mathrm{PO}_{4}, 150 \mathrm{mM} \mathrm{NaCl}[\mathrm{pH} 7.4], 0.1 \%$ Nonidet P-40, $0.5 \%$ sodium deoxycholate, $0.1 \%$ SDS) containing $10 \mu \mathrm{g} /$ $\mathrm{mL}$ phenylmethyl sulfonyl fluoride and $1 \mu \mathrm{g} / \mathrm{mL}$ aprotinin as protease inhibitor. The antibodies used for immunoprecipitation were rabbit anti-3-nitrotyrosine, rabbit antimTOR, or rabbit anti-Rictor. One mg of protein from whole cell lysate was incubated overnight with $2 \mu \mathrm{g}$ of corresponding antibody at $4{ }^{\circ} \mathrm{C}$ with continuous rotation, at which point $20 \mu \mathrm{L}$ of protein A/G beads (Santa Cruz, CA) were added to the reaction mixture and the rotation continued for another $2 \mathrm{~h}$ at $4{ }^{\circ} \mathrm{C}$. Immunoprecipitates were collected by centrifugation at $2500 \times g$ for $5 \mathrm{~min}$ followed by washing with binding buffers. After the final wash, all the adhering liquids were removed from the beads. Samples were then suspended in $1 \times$ Laemmli buffer, subjected to SDS-polyacrylamide gel electrophoresis, and specific proteins were detected by western blotting.

\section{Quantification of ROS levels by flow cytometry}

MitoSox Red (Thermo Scientific, Cat. M36008), a highly selective mitochondrial superoxide indicator for live cells, was used to measure ROS levels. Rotenone $(200 \mathrm{nM}$, Sigma), known to be a mitochondrial superoxide inducer, was used as a positive control. To account for superoxidespecific fluorescence, the cells were pretreated with 100 units/mL PEG-SOD (Sigma) or $20 \mu \mathrm{M} \mathrm{MnP}$ for $24 \mathrm{~h}$ prior to measurement. In brief, cells were loaded with $5 \mu \mathrm{M}$ MitoSox Red for $10 \mathrm{~min}$ at $37^{\circ} \mathrm{C}$ and rinsed three times with HBSS and then collected by trypsinization. Cell suspension (final volume of $500 \mu \mathrm{L}$ in $1 \times$ PBS) was analyzed using a BD FACS LSR II flow cytometer (Becton Dickinson). Ten thousand cells were acquired for each sample using the FACS DIVA software (Becton Dickinson). The results were then analyzed using Cell Quest Pro software.

\section{BrdU incorporation and detection assay}

Cell proliferation was assessed based on 5-bromo-2'-deoxyuridine (BrdU) incorporation followed by ELISA according to the manufacturer's protocol (Cell Biolabs, Inc.). Briefly, 30,000 cells were grown in a 96-well plate for $24 \mathrm{~h}$ at $37{ }^{\circ} \mathrm{C}$ in a $5 \% \mathrm{CO}_{2}$ incubator. BrdU solutions were added to the well to achieve $1 \mathrm{nM}$ final concentration and incubated for $6 \mathrm{~h}$. Cells were then washed, fixed, and denatured at $37{ }^{\circ} \mathrm{C}$ for $30 \mathrm{~min}$. Following successive washings with PBS, cellular BrdU uptake was detected by colorimetric ELISA $(450 \mathrm{~nm})$ using anti-BrdU antibody. Data were normalized to the protein concentration of the corresponding sample. 


\section{Statistical analysis}

Data were analyzed using one-way analysis of variance for multiple-group comparisons, and Student's $t$ test for twogroup comparisons. For multiple-group statistics, Bonferroni's post-test for multiple comparisons was used to determine the statistical significance.

Acknowledgements This work is supported, in part, by National Institutes of Health Grants CA 49797 and CA 73599 to Daret K. St. Clair, and the NCI Cancer Center Support Grant P30 CA177558 to B. Mark Evers.

\section{Compliance with ethical standards}

Conflict of interest The authors declare that they have no conflict of interest.

Open Access This article is licensed under a Creative Commons Attribution 4.0 International License, which permits use, sharing, adaptation, distribution and reproduction in any medium or format, as long as you give appropriate credit to the original author(s) and the source, provide a link to the Creative Commons license, and indicate if changes were made. The images or other third party material in this article are included in the article's Creative Commons license, unless indicated otherwise in a credit line to the material. If material is not included in the article's Creative Commons license and your intended use is not permitted by statutory regulation or exceeds the permitted use, you will need to obtain permission directly from the copyright holder. To view a copy of this license, visit http://creativecommons. org/licenses/by/4.0/.

\section{References}

1. Bogenhagen DF, Pinz KG, Perez-Jannotti RM. Enzymology of mitochondrial base excision repair. Prog Nucleic Acid Res Mol Biol. 2001;68:257-71.

2. Bolden A, Noy GP, Weissbach A. DNA polymerase of mitochondria is a gamma-polymerase. J Biol Chem. 1977;252:3351-6.

3. Hubscher U, Kuenzle CC, Spadari S. Functional roles of DNA polymerase beta and gamma. Proc Natl Acad Sci USA. 1976;76:2316-20.

4. Kaguni LS. DNA polymerase gamma, the mitochondrial replicase. Annu Rev Biochem. 2004;73:293-320.

5. Longley MJ, Prasad R, Srivastava DK, Wilson SH, Copeland WC. Identification of 5'deoxyribose phosphate lyase activity in human DNA polymerase gamma and its role in mitochondrial base excision repair in vitro. Proc Natl Acad Sci USA. 1998;95:12244-8.

6. Hance N, Ekstrand MI, Trifunovic A. Mitochondrial DNA polymerase gamma is essential for mammalian embryogenesis. Hum Mol Genet. 2005;14:1775-83.

7. Smeitink J, Van-den Heuvel L, DiMauro S. The genetics and pathology of oxidative phosphorylation. Nat Rev Genet. 2001;2:342-52.

8. Taylor RW, Turnbull DM. Mitochondrial DNA mutations in human disease. Nat Rev Genet. 2005;6:389-402.

9. Bakthavatchalu V, Dey S, Xu Y, Noel T, Jungsuwadee P, Holley $\mathrm{AK}$, et al. Manganese superoxide dismutase is a mitochondrial fidelity protein that protects Pol $\gamma$ against UV-induced inactivation. Oncogene. 2012;31:2129-39.

10. Alvarez B, Radi R. Peroxynitrite reactivity with amino acids and proteins. Amino Acids. 2003;25:295-311.
11. Graziewicz MA, Day BJ, Copeland WC. The mitochondrial DNA polymerase as a target of oxidative damage. Nucleic Acids Res. 2002;30:2817-24

12. Rodriguez-Rocha H, Garcia-Garcia A, Panayiotidis M, Franco R. DNA damage and autophagy. Mutat Res. 2011;711:158-66.

13. Abada A, Elazar Z. Getting ready for building: signaling and autophagosome biogenesis. EMBO Rep. 2014;15:839-52.

14. Boya P, Reggiori F, Codogno P. Emerging regulation and functions of autophagy. Nat Cell Biol. 2013;15:713-20.

15. Choi AM, Ryter SW, Levine B. Autophagy in human health and disease. New Engl J Med. 2013;368:651-62.

16. Vander Heiden MG, Cantley LC, Thompson CB. Understanding the Warburg effect: the metabolic requirements of cell proliferation. Science. 2009;324:1029-33.

17. Gonzalez DHM, Paz ML, Ferrari A, Weill FS, Czerniczyniec A, Leoni J, et al. Skin damage and mitochondrial dysfunction after acute ultraviolet $\mathrm{B}$ irradiation: relationship with nitric oxide production. Photodermatol Photoimmunol Photomed. 2005;21:311-7.

18. Wu S, Wang L, Jacoby AM, Jasinski K, Kubant R, Malinski T. Ultraviolet B light-induced nitric oxide/peroxynitrite imbalance in keratinocytes-implications for apoptosis and necrosis. Photochem Photobiol. 2010;86:389-96.

19. Pettersson AS, Steen H, Kalume DE, Caidahl K, Roepstorff P. Investigation of tyrosine nitration in proteins by mass spectrometry. J Mass Spectrom. 2001;36:616-25.

20. Copeland WC, Longley MJ. DNA polymerase $\gamma$. Sci World J. 2003;3:34-44.

21. Kovalenko OA, Santos JH. Analysis of oxidative damage by gene-specific quantitative PCR. Curr Protoc Hum Genet. 2009; S62:19.1.1-19.1.13.

22. Tanida I, Ueno T, Kominami E. LC3 conjugation system in mammalian autophagy. Int J Biochem Cell Biol. 2004;36:2503-18.

23. Aoki H, Kondo Y, Aldape K, Yamamoto A, Iwado E, Yokoyama $\mathrm{T}$, et al. Monitoring autophagy in glioblastoma with antibody against isoform B of human microtuble-associated protein 1 light chain 3. Autophagy. 2008;4:467-75.

24. He C, Klionsky DJ. Regulation mechanisms and signaling pathways of autophagy. Annu Rev Genet. 2009;43:67-93.

25. Levine B, Kroemer G. Autophagy in the pathogenesis of disease. Cell. 2008;132:27-42.

26. Wang RC, Wei Y, An Z, Zou Z, Xiao G, Bhagat G, et al. Aktmediated regulation of autophagy and tumorigenesis through beclin 1 phosphorylation. Science. 2012;338:956-9.

27. Sabatini DM. mTOR and cancer: insights into a complex relationship. Nat Rev. 2006;6:729-34.

28. MacMillan-Crow L,Thompson JA, Tyrosine modifications and inactivation of active site manganese superoxide dismutase mutant (Y34F) by peroxynitrite. Arch Biochem Biophy. 1999;366:82-8.

29. Hausladen A, Fridovich I. Superoxide and peroxynitrite inactivate aconitases, nitric oxide does not. J Biol Chem. 1994;269:29405-8.

30. Matsuzawa Y, Oshima S, Takahara M, Maeyashiki C, Nemoto Y, Kobayashi M, et al. TNFAIP3 promotes survival of CD4 T cells by restricting mTOR and promoting autophagy. Autophagy. 2015;11:1052-62.

31. Dang A, Huang C, Tandon A, Stolz D, Wu T, Gandhi CR. Endotoxin-stimulated rat hepatic stellate cells induce autophagy in hepatocytes as a survival mechanism. J Cell Physiol. 2016;231:94-105.

32. Duan H, Li Y, Yan L, Yang H, Wu J, Qian P, et al. Rcan1-1L overexpression induces mitochondrial autophagy and improves cell survival in angiotensin II-exposed cardiomyocytes. Exp Cell Res. 2015;335:99-106.

33. Laplante M, Sabatini DM. mTOR signaling in growth control and disease. Cell. 2012;149:274-93.

34. Chang HJ, Seung-Hyun R, Jing C, Neil MO, Do-Hyung K. mToR regulation of autophagy. FEBS Lett. 2010;584:1287-95. 
35. Wang P, Guo QS, Wang ZW, Qian HX. HBx induces HepG-2 cells autophagy through PI3K/Akt-mTOR pathway. Mol Cell Biochem. 2013;372:161-8.

36. Noda T, Ohsumi Y. TOR, a phosphatidylinositol kinase homologue, controls autophagy in yeast. $\mathrm{J}$ Biol Chem. 1998;273:3963-6.

37. Matsuda-Lennikov M, Suizu F, Hirata N, Hashimoto M, Kimura K, Nagamine T, et al. Lysosomal interaction of Akt with Phafin2: a critical step in the induction of autophagy. PLoS ONE. 2014;9: e79795.

38. Pua HH, Guo J, Komatsu M, He Y-W. Autophagy is essential for mitochondrial clearance in mature $\mathrm{T}$ lymphocytes. J Immun. 2009;182:4046-55.

39. Fan Q-W, Weiss WA. Autophagy and Akt promote survival in glioma. Autophagy. 2011;7:536-8.

40. Dhar SK, Lynn BC, Daosukho C, St. Clair DK. Identification of nucleophosmin as an NF- $\mathrm{KB}$ co-activator for the induction of human SOD2 gene. J Biol Chem. 2004;279:28209-19.

41. Dhar SK, Xu Y, St. Clair DK. Nuclear factor kappaB and specificity protein 1-dependent bi-directional regulation of human manganese superoxide dismutase. J Biol Chem. 2010; 284:9835-46.

42. Ran FA, Hsu PD, Wright J, Agarwala V, Scott DA, Zhang F. Genome engineering using the CRISPR-Cas9 system. Nat Protoc. 2013;8:2281-308

43. Dhar SK, Zhang J, Gal J, Xu Y, Miao L, Lynn BC, et al. Fused in sarcoma is a novel regulator of manganese superoxide dismutase gene transcription. Antioxid Redox Signal. 2014;20:1550-66.

44. Longley MJ, Copeland WC. Purification, separation, and identification of the human mtDNA polymerase with and without its accessory subunit. Methods Mol Biol. 2002;197:245-57.

45. Szczesny B, Olah G, Walker DK, Volpi E, Rasmussen BB, Szabo $\mathrm{C}$, et al. Deficiency in repair of the mitochondrial genome sensitizes proliferating myoblast to oxidative damage. PLoS ONE. 2013;8:e75201.

46. Shi J, Wang Y, Zeng L, Wu Y, Deng J, Zhang Q, et al. Disrupting the interaction of BRD4 with diacetylated Twist suppresses tumorigenesis in basal-like breast cancer. Cancer Cell. 2014;25:210-25. 